Discussion Paper Series A No.594

\title{
Unequal Exchange, Assets, and Power: Recent \\ Developments in Exploitation Theory
}

\author{
Roberto Veneziani \\ (School of Economics and Finance, Queen Mary University of London) \\ and \\ Naoki Yoshihara \\ (The Institute of Economic Research, Hitotsubashi University)
}

September, 2013

Institute of Economic Research

Hitotsubashi University

Kunitachi, Tokyo, 186-8603 Japan 


\title{
Unequal exchange, assets, and power: recent developments in exploitation theory*
}

\author{
Roberto Veneziani ${ }^{\dagger}$ and Naoki Yoshihara ${ }^{\ddagger}$
}

September 7, 2013

\begin{abstract}
This paper surveys and extends some recent contributions on the theory of exploitation as the unequal exchange of labour. A model of dynamic economies with heterogeneous optimising agents is presented which encompasses the models used in the literature as special cases. It is shown that the notion of exploitation is logically coherent and can be meaningfully analysed in such a general framework. It is then shown that the axiomatic approach of social choice theory can be adopted to explore the normative foundations of the notion of exploitation. Finally, it is argued that purely distributive approaches to exploitation are not entirely compelling and a notion of dominance, or unequal power is necessary.
\end{abstract}

JEL classification: D63; D51.

Keywords: Exploitation, axiomatic social choice.

${ }^{*}$ A preliminary version of the paper was presented at CEPET 2010 Workshop in honour of Nick Baigent, where Nick Baigent, Constanze Binder, Giulio Codognato, Rajat Deb, and Yongsheng Xu provided insightful and useful comments. We are also grateful to Meghnad Desai, Woojin Lee, John Roemer, Ian Steedman, and participants in the "Capitalism, Socialism and Democracy" conference (Amherst), the MANCEPT Workshops and seminars at the LSE, the University of Siena, and the Institute of Education (London) for comments and suggestions on an earlier version of this paper. Special thanks go to Gil Skillman, the editors and two anonymous referees for long and detailed comments. The usual disclaimer applies.

${ }^{\dagger}$ School of Economics and Finance, Queen Mary University of London, Mile End Road, London E1 4NS, UK; and Department of Economics, University of Massachusetts, Amherst. E-mail: r.veneziani@qmul.ac.uk

$\ddagger$ (Corresponding author) The Institute of Economic Research, Hitotsubashi University, Naka 2-1, Kunitachi, Tokyo 186-0004, Japan. Phone: (81)-42-580-8354. E-mail: yosihara@ier.hit-u.ac.jp 


\section{Introduction}

The notion of exploitation is prominent in the social sciences and in political philosophy. It is central in Marxist-based analyses of labour relations but it is also extensively discussed in liberal approaches, especially in the analysis of (possibly mutually beneficial) trades characterised by significant disparities in bargaining power. ${ }^{1}$ Yet, it has received relatively little attention in social choice theory and in normative economics. This is due partly to the traditional association of exploitation theory with the labour theory of value, whose logical flaws are assumed to carry over to the notion of exploitation, and to the fact that exploitation is usually analysed under fairly restrictive assumptions concerning technology, preferences, and endowments. But it is also due to the focus of social choice theory on distributive issues, and more specifically on the distribution of welfare, income, wealth, resources, or more recently, capabilities and opportunities.

John Roemer's classic work [24], [25], [26] has demonstrated that a coherent notion of exploitation can be provided independently of the labour theory of value. Moreover, he has proved that at least some of the key insights of exploitation theory hold outside of simple Leontief economies with homogeneous labour, subsistence consumption, and a polarised class structure. Somewhat paradoxically, however, the operation succeeded, but the patient died: the main conclusion of Roemer's work is that a concern for asset inequalities is the only sound legacy of exploitation theory, which reduces to a variant of liberal egalitarianism and is 'a domicile that we need no longer maintain: it has provided a home for raising a vigorous family, who now must move on' (Roemer [28]: 67).

This paper surveys and extends recent work in exploitation theory and argues that the concept of exploitation is logically and theoretically sound, and provides interesting normative insights on the wrongs that characterise advanced capitalist economies, which go beyond the standard distributive focus of social choice theory and normative economics.

First, exploitation can be rigorously analysed in a rather general framework. Section 2 sets up a model of a dynamic economy with a convex technology, and heterogeneous optimising agents endowed with different amounts of physical and human capital. We discuss both the individual maximisation

\footnotetext{
${ }^{1}$ The literature is too vast for a comprehensive list of references, but recent contributions include van Donselaar [33], Ypi [45], Fleurbaey [10], Steiner [32], Vrousalis [38].
} 
programme and the equilibrium notion - the concept of Reproducible Solution proposed by Roemer [23], [24], - and show that the structure of the economy is similar to standard growth models. Then we show that, contrary to the received view, the standard static models used in the literature are not ad hoc and can be interpreted as focusing on the steady state equilibria of the general model.

Second, unlike in the main theories of distributive justice, exploitation focuses on labour as the variable of normative interest. In the theory of exploitation as an unequal exchange (UE) of labour, exploitative relations are characterised by systematic differences between the amount of labour that individuals contribute to the economy, in some relevant sense, and the amount of labour they receive, in some relevant sense, via their income.

We argue that the key normative insights of the notion of exploitation can be captured within the rigorous axiomatic framework of social choice theory. An axiomatic approach to exploitation was long overdue: outside of simple stylised economies, many definitions can be, and have in fact been proposed that incorporate different positive and normative intuitions. By adopting an axiomatic method, we start from first principles, thus explicitly discussing the intuitions underlying UE exploitation. Moreover, an axiomatic approach demonstrates that the notion of exploitation is not obscure or incoherent, and relies on some theoretically robust and normatively relevant intuitions that can be precisely stated in the rigorous language of normative economics.

Section 3 discusses some recent axiomatic analyses of exploitation theory. In particular, we analyse a characterisation of the class of UE exploitationforms as indicators of capitalist relations of production that allow wealthy agents to appropriate social surplus generated from social labour as profits. Our characterisation result provides the necessary and sufficient condition for coherent definitions of exploitation, in that the basic property of exploitation and profits holds regardless of the complexity of the economic models. This characterisation leads us to conclude that among main approaches, an extension of the 'New Interpretation' form of exploitation (Duménil [5], [6]; Foley [11], [12]) is the only coherent definition in this respect.

Another contribution of the paper is to argue that, unlike most of normative economics and social choice, the notion of exploitation suggests that the wrongs of capitalist economies go beyond inequalities in economic outcomes or opportunities. As Roemer [24], [26], [28] has forcefully argued, distributive injustices are at the core of exploitative relations and theories of exploitation based on dominance in the workplace or coercion in the labour market 
are unsatisfactory. However, at the philosophical level, purely distributive approaches - such as Roemer's - have too impoverished an informational basis to capture exploitative relations and to distinguish exploitation from other forms of injustice, or wrongs. Some notion of power, or dominance, or asymmetric relations between agents is an essential - definitional - part of exploitation, and this emphasis on the structure of the interaction between agents that allows someone to take (unfair) advantage of somebody else is an important contribution of exploitation theory that may correct the 'distributive bias' of normative economics. In this respect, we take inspiration from some seminal contributions by Nick Baigent [1], which explore rights and more generally non-consequentialist principles in social choice.

Based on the general model set up in section 2, section 4 builds on and extends some recent contributions that analyse exploitation in a dynamic context. It is shown that inequalities in productive assets are not sufficient for exploitation to provide foundations to exploitation as a persistent phenomenon. Something else is necessary in order to generate persistent exploitation, and power or dominance are natural candidates for that role.

\section{The general model}

This section sets up the model and the relevant equilibrium notion. Compared to the standard literature in exploitation theory and mathematical Marxian economics, our economies are general in at least three key dimensions. First, as in Roemer [23], we allow for a general convex cone production set, rather than the canonical Leontief or von Neumann technology. As is well known (Morishima [18],[19]), outside of the simple linear production model, many of the classical Marxian propositions do not necessarily hold: there is no obvious way of defining the labour value of each commodity; it is not clear that the two aggregate equalities between the sum of prices and the sum of values, and the sum of profits and the sum of surplus values can simultaneously hold; and so on. We aim to show that a logically consistent and theoretically rigorous notion of UE exploitation can instead be provided even in general production economies. This is essential in order to defend the normative relevance of UE in advanced capitalist economies.

Second, unlike in the standard literature, we do not focus on polarised, two-class economies in which capitalists save and accumulate while workers spend their wage revenue to buy a fixed subsistence bundle. Rather, we 
allow for agent heterogeneity concerning endowments of physical assets, as in Roemer [23], [24], and also for heterogeneous preferences and human capital. Further, rather than assuming individuals to belong to given classes, our general models allow one to analyse the class structures that endogenously emerge in the equilibrium of economies in which agents are allowed to save and thus class mobility is not ruled out.

In fact, third, we take account of the dynamic structure of the economy. On the one hand, as in Roemer [23], [24], we explicitly incorporate the time structure of production processes - whereby production takes time and outputs emerge only at the end of a given production period - and the fact that capital goods are reproducible. This is a major difference with standard neoclassical models which usually ignore the time structure of production, as in Walrasian general equilibrium theory, or treat capital as a primary factor, as in the Heckscher-Ohlin-Samuelson theory of international trade. The former feature implies that the role of capital scarcity in generating exploitation and classes cannot be analysed, whereas the latter feature yields a theory of profit that is analogous to the theory of rent.

On the other hand, unlike in the classic literature, including Roemer's seminal contributions, we explicitly model competitive resource allocations as involving a dynamic structure of economic interactions and assume that individuals face an intertemporal optimisation programme. We provide a definition of equilibrium in this dynamic setting that generalises Roemer's [23], [24] static notion of reproducible solution and show that the latter is a temporary equilibrium notion which can be interpreted as a one-period feature of our general equilibrium concept.

\subsection{Technology}

An economy comprises a set of agents $\mathcal{N}=\{1, . ., N\}$. A sequence of nonoverlapping generations exist, each living for $T$ periods, where $T$ can be either finite or infinite, and indexed by the date of birth $k T, k=0,1,2, \ldots$ Let $\mathbb{R}$ be the set of real numbers and let $\mathbb{R}_{+}, \mathbb{R}_{-}$be, respectively, the set of nonnegative and nonpositive real numbers.

Production technology is freely available to all agents, who can operate any activity in the production set $P$, which has elements of the form $\alpha=$ $\left(-\alpha_{l},-\underline{\alpha}, \bar{\alpha}\right)$ where $\alpha_{l} \in \mathbb{R}_{+}$is the effective labour input; $\underline{\alpha} \in \mathbb{R}_{+}^{n}$ are the inputs of the produced goods; and $\bar{\alpha} \in \mathbb{R}_{+}^{n}$ are the outputs of the $n$ goods. Thus, elements of $P$ are vectors in $\mathbb{R}^{2 n+1}$. The net output vector arising 
from $\alpha$ is denoted as $\widehat{\alpha} \equiv \bar{\alpha}-\underline{\alpha}$. Let $\mathbf{0}$ denote the null vector. The following assumptions on $P$ hold throughout the paper. ${ }^{2}$

Assumption 0 (A0). $P$ is a closed convex cone in $\mathbb{R}^{2 n+1}$ and $\mathbf{0} \in P$.

Assumption 1 (A1). For all $\alpha \in P, \bar{\alpha} \geq \mathbf{0} \Rightarrow \alpha_{l}>0$.

Assumption 2 (A2). For all $c \in \mathbb{R}_{+}^{n}, \exists \alpha \in P: \widehat{\alpha} \geqq c$.

Assumption 3 (A3). For all $\alpha \in P$ and all $\alpha^{\prime} \in \mathbb{R}_{-} \times \mathbb{R}_{-}^{n} \times \mathbb{R}_{+}^{n},\left[\alpha^{\prime} \leqq \alpha \Rightarrow\right.$ $\left.\alpha^{\prime} \in P\right]$.

A1 implies that labour is indispensable to produce any output. A2 states that any non-negative commodity vector is producible as net output. A3 is a standard free disposal condition.

A0 $\mathbf{A 3}$ are quite general and include the standard production technologies discussed in mathematical Marxian economics as special cases. For example, the Leontief technology with a $n \times n$ non-negative input matrix $A$ and a $1 \times n$ positive vector of labour inputs $L$ is represented by

$$
P_{(A, L)} \equiv\left\{\alpha \in \mathbb{R}_{-} \times \mathbb{R}_{-}^{n} \times \mathbb{R}_{+}^{n} \mid \exists x \in \mathbb{R}_{+}^{n}: \alpha \leqq(-L x,-A x, x)\right\} .
$$

Given $P$, the set of activities feasible with $k$ units of effective labour is:

$$
P\left(\alpha_{l}=k\right) \equiv\left\{\left(-\alpha_{l},-\underline{\alpha}, \bar{\alpha}\right) \in P \mid \alpha_{l}=k\right\} ;
$$

$\partial P \equiv\left\{\alpha \in P \mid \nexists \alpha^{\prime} \in P: \alpha^{\prime}>\alpha\right\}$ is the frontier of $P$; and for any $c \in \mathbb{R}_{+}^{n}$ ， the set of activities that produce at least $c$ as net output is:

$$
\phi(c) \equiv\{\alpha \in P \mid \widehat{\alpha} \geqq c\} .
$$

\subsection{Agents}

In the economy, agents produce, consume, and trade labour. On the production side, they can either sell their labour-power or hire workers to work on their capital, or they can be self-employed and work on their own assets.

In every period $t,\left(p_{t}, w_{t}\right) \in \mathbb{R}_{+}^{n+1} \backslash\{\mathbf{0}\}$ denotes the $1 \times(n+1)$ price vector that prevails in competitive markets. Let $\Delta \equiv\left\{(p, w) \in \mathbb{R}_{+}^{n+1} \mid \sum_{i=1}^{n} p_{i}+w=1\right\}$.

For all $\nu \in \mathcal{N}$, let $\varsigma^{\nu}>0$ be agent $\nu$ 's skill level. Then, for all $\nu \in \mathcal{N}$, in every $t$ : $\alpha_{t}^{\nu}=\left(-\alpha_{l t}^{\nu},-\underline{\alpha}_{t}^{\nu}, \bar{\alpha}_{t}^{\nu}\right) \in P$ is the production process operated

\footnotetext{
${ }^{2}$ For all $x, y \in \mathbb{R}^{n}, x \geqq y$ if and only if $x_{i} \geqq y_{i}(i=1, \ldots, n) ; x \geq y$ if and only if $x \geqq y$ and $x \neq y ; x>y$ if and only if $x_{i}>y_{i}(i=1, \ldots, n)$.
} 
with $\nu$ 's own capital and labour, where $\alpha_{l t}^{\nu}=\varsigma^{\nu} a_{l t}^{\nu}$ and $a_{l t}^{\nu}$ is the labour time expended by $\nu ; \beta_{t}^{\nu}=\left(-\beta_{l t}^{\nu},-\underline{\beta}_{t}^{\nu}, \bar{\beta}_{t}^{\nu}\right) \in P$ is the production process operated by hiring others; $\gamma_{t}^{\nu}=\varsigma^{\nu} l_{t}^{\nu}$ is $\nu$ 's effective labour supply, where $l_{t}^{\nu}$ is the labour time supplied by $\nu$ on the market. At any $t, \lambda_{t}^{\nu}=\left(a_{l t}^{\nu}+l_{t}^{\nu}\right)$ is the total amount of labour time expended by $\nu$ and $\Lambda_{t}^{\nu}=\alpha_{l t}^{\nu}+\gamma_{t}^{\nu}=\varsigma^{\nu} \lambda_{t}^{\nu}$ is the total amount of effective labour performed by $\nu$, either as a self-employed producer or working for some other agent. Further, for all $\nu \in \mathcal{N}, s_{t}^{\nu} \in \mathbb{R}^{n}$ is the vector of net savings and $\omega_{t}^{\nu} \in \mathbb{R}_{+}^{n}$ is the vector of productive endowments, where $\omega_{k T}^{\nu}$ denotes the endowments inherited when born in $k T$.

As in Roemer [23], [24], the time structure of production is explicitly considered and production activities are financed with current wealth. Agent $\nu$ 's wealth, at the beginning of $t$, is given by $W_{t}^{\nu}=p_{t-1} \omega_{t}^{\nu}$ : this is fixed at the end of $t-1$ given previous savings decisions $s_{t-1}$ and market prices $p_{t-1}$. At the beginning of $t, \nu$ uses $W_{t}^{\nu}$ to purchase a vector of capital goods $\underline{\alpha}_{t}^{\nu}+\underline{\beta}_{t}^{\nu}$ at prices $p_{t-1}$ and any wealth left can be used to purchase a vector of goods $\delta_{t}^{\nu} \in \mathbb{R}_{+}^{n}$ that can be sold on the market at the end of $t$.

On the consumption side, for each agent $\nu: C \subseteq \mathbb{R}_{+}^{n}$ is the consumption set, $c_{t}^{\nu} \in C$ is the consumption vector at $t$, and total labour hours expended cannot exceed the endowment which is normalised to one. Agent $\nu$ 's welfare is given by a monotonic function $u^{\nu}: C \times[0,1] \rightarrow \mathbb{R}_{+}$, which is increasing in consumption and decreasing in labour time.

For any $t$, let $\Omega_{t}=\left(\omega_{t}^{1}, \omega_{t}^{2}, \ldots, \omega_{t}^{N}\right) ; E\left(P, \mathcal{N}, C,\left(u^{\nu}\right)_{\nu \in \mathcal{N}},\left(\varsigma^{\nu}\right)_{\nu \in \mathcal{N}}, \Omega_{k T}\right)$ denotes the economy with technology $P$, agents $\mathcal{N}$, consumption set $C$, welfare functions $\left(u^{\nu}\right)_{\nu \in \mathcal{N}}$, skills $\left(\varsigma^{\nu}\right)_{\nu \in \mathcal{N}}$, and productive endowments $\Omega_{k T}$. The universal class of all such convex cone economies is $\mathcal{E}$.

Let $c^{\nu}=\left\{c_{t}^{\nu}\right\}_{t=k T}^{(k+1) T-1}$ be $\nu$ 's lifetime consumption plan; and likewise for $\alpha^{\nu}, \beta^{\nu}, \gamma^{\nu}, \delta^{\nu}, s^{\nu}$, and $\omega^{\nu}$. Let $(\mathbf{p}, \mathbf{w})=\left\{\left(p_{t}, w_{t}\right)\right\}_{t=k T}^{(k+1) T-1}$ be the path of price vectors during the lifetime of a generation. Let $\xi^{\nu}=\left(\alpha^{\nu}, \beta^{\nu}, \gamma^{\nu}, \delta^{\nu}, c^{\nu}, s^{\nu}\right)$ denote a generic intertemporal plan for $\nu$, with $\xi_{t}^{\nu}=\left(\alpha_{t}^{\nu}, \beta_{t}^{\nu}, \gamma_{t}^{\nu}, \delta_{t}^{\nu}, c_{t}^{\nu}, s_{t}^{\nu}\right)$ at any $t$. Let $0<\rho \leq 1$ be the time preference factor. Given $(\mathbf{p}, \mathbf{w})$, each agent $\nu$ chooses $\xi^{\nu}$ to maximise welfare subject to the constraint that in every $t$ : (1) income is sufficient for consumption and savings; (3) production activities, consumption choices and labour performed are feasible; and (4) the dynamics of capital is determined by net savings. Furthermore, (2) wealth must be sufficient for production plans and any wealth not used productively is carried over to the end of the period. Finally, (5) reproducibility requires resources not to be depleted; in particular, generation $k$ is constrained to 
bequeath at least as many resources as they inherited. Formally:

$$
M P^{\nu}: V\left(\omega_{k T}^{\nu}\right)=\max _{\xi^{\nu}} \sum_{t=k T}^{(k+1) T-1} \rho^{t} u^{\nu}\left(c_{t}^{\nu}, \lambda_{t}^{\nu}\right),
$$

subject to (for all $t=k T, \ldots,(k+1) T-1)$ :

$$
\begin{aligned}
{\left[p_{t} \bar{\alpha}_{t}^{\nu}\right]+\left[p_{t} \bar{\beta}_{t}^{\nu}-w_{t} \beta_{l t}^{\nu}\right]+w_{t} \gamma_{t}^{\nu}+p_{t} \delta_{t}^{\nu} } & \geqq p_{t} c_{t}^{\nu}+p_{t} \omega_{t+1}^{\nu} \\
p_{t-1} \delta_{t}^{\nu}+p_{t-1}\left(\underline{\alpha}_{t}^{\nu}+\underline{\beta}_{t}^{\nu}\right) & =p_{t-1} \omega_{t}^{\nu} \\
\alpha_{t}^{\nu}, \beta_{t}^{\nu} & \in P,\left(c_{t}^{\nu}, \lambda_{t}^{\nu}\right) \in C \times[0,1] \\
\omega_{t+1}^{\nu} & =\omega_{t}^{\nu}+s_{t}^{\nu} \\
\omega_{(k+1) T}^{\nu} & \geqq \omega_{k T}^{\nu} .
\end{aligned}
$$

$M P^{\nu}$ generalises similar programmes in Roemer [23], [24]. As in standard microeconomics, agents are not assumed to be 'agents of capital' or to produce for production's own sake: they are endowed with general preferences over consumption and leisure. However, following Roemer [23], [24], and unlike in the standard approach, $M P^{\nu}$ explicitly incorporates the simultaneous role of economic actors as consumers and producers - so that no separate consideration of firms is necessary, - and the time structure of the production process. Thus, at the beginning of each $t$, agent $\nu$ supplies $\gamma_{t}^{\nu}$ on the labour market and uses her wealth $W_{t}^{\nu}$ to purchase goods $\underline{\alpha}_{t}^{\nu}+\beta_{t}^{\nu}+\delta_{t}^{\nu}$ at prices $p_{t-1}$. The capital goods $\underline{\alpha}_{t}^{\nu}+\underline{\beta}_{t}^{\nu}$ are used to activate production by employing $\beta_{l t}^{\nu}$ units of labour, whereas $\delta_{t}^{\nu}$ are carried over to the end of the period. Production then takes place and outputs appear at the end of $t$, when $\nu$ 's proceedings from production are $p_{t}\left(\bar{\alpha}_{t}^{\nu}+\bar{\beta}_{t}^{\nu}\right)$ and wage earnings are $w_{t} \gamma_{t}^{\nu}$. Therefore, gross revenue at $t$ is $p_{t}\left(\bar{\alpha}_{t}^{\nu}+\bar{\beta}_{t}^{\nu}\right)+w_{t} \gamma_{t}^{\nu}+p_{t} \delta_{t}^{\nu}$ which is used to pay $w_{t} \beta_{l t}^{\nu}$ to employees, and to purchase - at the current prices $p_{t}$ - consumption goods $c_{t}^{\nu}$ and capital goods $\omega_{t+1}^{\nu}=\omega_{t}^{\nu}+s_{t}^{\nu}$ for next period's production.

Agents need to lay out in advance the capital necessary for production and can do so only by using their own wealth, which may be deemed restrictive. Two points should be noted here. First, as in Roemer [24], [26], this assumption rules out intertemporal credit markets and intertemporal trade between agents. Due to the possibility of saving, however, the model allows for intertemporal trade-offs in the allocation of labour and consumption goods 
during an agent's life, consistently with a dynamic setting in which agents' lives are divided into more than one period and this significantly generalises Roemer's models. Second, a credit market may be introduced but it would not change the main results (see Roemer [23], chapter 3; [24]).

Finally, our conclusions are robust to alternative specifications of the individual optimisation programme. All of the main insights continue to hold if $M P^{\nu}$ is reformulated by focusing on end-of-period prices $p_{t}$ in (2), which generalises Veneziani [34]; or by letting the length of the production period tend to zero, so as to move to a continuous time setting, as in Veneziani [35].

\subsection{Equilibrium}

Let $c_{t}=\sum_{\nu=1}^{N} c_{t}^{\nu}$; and likewise for all other variables. For the sake of simplicity, let 'all $t$ ' stand for 'all $t=k T, \ldots,(k+1) T-1$ '. Let $\mathcal{O}^{\nu}(\mathbf{p}, \mathbf{w}) \equiv$ $\left\{\xi^{\nu}\right.$ solves $M P^{\nu}$ at $\left.(\mathbf{p}, \mathbf{w})\right\}$. The equilibrium concept can now be defined.

Definition 1 A reproducible solution $(R S)$ for $E\left(P, \mathcal{N}, C,\left(u^{\nu}\right)_{\nu \in \mathcal{N}},\left(\varsigma^{\nu}\right)_{\nu \in \mathcal{N}}, \Omega_{k T}\right)$ is a price vector $(\mathbf{p}, \mathbf{w})$ and an associated set of actions such that:

(i) $\xi^{\nu} \in \mathcal{O}^{\nu}(\mathbf{p}, \mathbf{w})$, all $\nu$;

(ii) $\widehat{\alpha}_{t}+\widehat{\beta}_{t} \geqq c_{t}+s_{t}$, all $t$;

(iii) $\underline{\alpha}_{t}+\underline{\beta}_{t}+\delta_{t} \leqq \omega_{t}$, all $t$;

(iv) $\beta_{l t}=\bar{\gamma}_{t}$, all $t$

(v) $\omega_{(k+1) T} \geqq \omega_{k T}$.

The equilibrium notion is standard. Condition (i) requires that every agent optimises. Conditions (ii) and (iii) are aggregate excess demand requirements. The former states that in every $t$ there must be enough resources for consumption and saving plans, and it is equivalent to: $\bar{\alpha}_{t}+\bar{\beta}_{t}+\left(\omega_{t}-\underline{\alpha}_{t}-\underline{\beta}_{t}\right) \geqq$ $c_{t}+\left(\omega_{t}+s_{t}\right)$, which states that, at the end of period $t$, the aggregate supply of resources available be at least as big as the aggregate demand for consumption and investment goods. The latter states that demand should not exceed supply in the produced inputs market and in every $t$ there must be enough resources for production plans. Condition (iv) imposes labour market clearing in every $t$.

Condition (v) is the intertemporal reproducibility condition, which requires that every generation leave to the following at least as many resources as they have inherited. This significantly relaxes the analogous reproducibility condition implicit in Roemer's [23], [24] static models without savings in 
which $\omega_{t+1} \geqq \omega_{t}$ automatically follows from conditions (ii) and (iii). In a finite horizon model, condition ( $\mathrm{v}$ ) can be seen as a simple fairness and sustainability condition analogous to the constraints often imposed in optimal Ramsey growth problems (see, for example, Morishima [17], Chapter 13). Formally, this condition is consistent with the transversality condition which is necessary in an infinite horizon model.

In what follows, we devote special attention to the subset of stationary equilibria in which prices and actions remain constant over time:

Definition 2 A stationary reproducible solution $(S R S)$ for $E\left(P, \mathcal{N}, C,\left(u^{\nu}\right)_{\nu \in \mathcal{N}},\left(\varsigma^{\nu}\right)_{\nu \in \mathcal{N}}, \cdot\right)$ is a price vector $(\mathbf{p}, \mathbf{w})$, an associated set of actions $\left(\xi^{\nu}\right)_{\nu \in \mathcal{N}}$, and a profile of capital stocks $\Omega^{*}=\left(\omega^{* 1}, \omega^{* 2}, \ldots, \omega^{* N}\right)$ such that $\left\langle(\mathbf{p}, \mathbf{w}),\left(\xi^{\nu}\right)_{\nu \in \mathcal{N}}\right\rangle$ is a $R S$ for $E\left(P, \mathcal{N}, C,\left(u^{\nu}\right)_{\nu \in \mathcal{N}},\left(\varsigma^{\nu}\right)_{\nu \in \mathcal{N}}, \Omega^{*}\right)$ with:

(1) $\left(p_{t}, w_{t}\right)=\left(p_{t+1}, w_{t+1}\right)$, all $t$;

(2) for any $\nu \in \mathcal{N}, \xi^{\nu} \in \mathcal{O}^{\nu}(\mathbf{p}, \mathbf{w})$ is such that $\xi_{t}^{\nu}=\xi_{t+1}^{\nu}$ and $s_{t}^{\nu}=\mathbf{0}$, all $t$.

In order to analyse the existence and properties of SRSs, it suffices to consider a stationary price vector $(\mathbf{p}, \mathbf{w})$ with $\left(p_{t}, w_{t}\right)=\left(p_{t+1}, w_{t+1}\right)=(p, w)$ for all $t$. In this case, programme $M P^{\nu}$ reduces to the following:

$$
M P^{\nu}: V\left(\omega_{k T}^{\nu}\right)=\max _{\xi^{\nu}} \sum_{t=k T}^{(k+1) T-1} \rho^{t} u^{\nu}\left(c_{t}^{\nu}, \lambda_{t}^{\nu}\right),
$$

subject to (for all $t)$ :

$$
\begin{aligned}
{\left[p\left(\bar{\alpha}_{t}^{\nu}-\underline{\alpha}_{t}^{\nu}\right)\right]+\left[p\left(\bar{\beta}_{t}^{\nu}-\underline{\beta}_{t}^{\nu}\right)-w \beta_{l t}^{\nu}\right]+w \gamma_{t}^{\nu} } & \geqq p c_{t}^{\nu}+p s_{t}^{\nu} \\
p\left(\underline{\alpha}_{t}^{\nu}+\underline{\beta}_{t}^{\nu}\right) & \leqq p \omega_{t}^{\nu}, \\
\alpha_{t}^{\nu}, \beta_{t}^{\nu} & \in P,\left(c_{t}^{\nu}, \lambda_{t}^{\nu}\right) \in C \times[0,1], \\
\omega_{t+1}^{\nu} & =\omega_{t}^{\nu}+s_{t}^{\nu}, \\
\omega_{(k+1) T}^{\nu} & \geqq \omega_{k T}^{\nu} .
\end{aligned}
$$

Further, noting that at a SRS, $\max _{\alpha_{t}^{\prime} \in P} \frac{p \widehat{\alpha}_{t}^{\prime}-w \alpha_{l t}^{\prime}}{p \underline{\alpha}_{t}^{\prime}}=\frac{1-\rho}{\rho}$ all $t$, the set $\Delta(\rho) \equiv$ $\left\{\left(p^{\prime}, w^{\prime}\right) \in \Delta \mid p^{\prime}\left(\bar{\alpha}-\rho^{-1} \underline{\alpha}\right)-w^{\prime} \alpha_{l} \leqq 0\right.$ for all $\left.\alpha \in P\right\}$ is compact and convex. Then, for any given $(p, w) \in \Delta(\rho)$, the individual optimisation programme can be further reduced to the following:

$$
M P^{\nu}: \max _{\alpha^{\nu}, \beta^{\nu} \in P,\left(c^{\nu}, \lambda^{\nu}\right) \in C \times[0,1]} u^{\nu}\left(c^{\nu}, \lambda^{\nu}\right)
$$


subject to

$$
\begin{aligned}
{\left[p\left(\bar{\alpha}^{\nu}-\underline{\alpha}^{\nu}\right)\right]+\left[p\left(\bar{\beta}^{\nu}-\underline{\beta}^{\nu}\right)-w \beta_{l}^{\nu}\right]+w \gamma^{\nu} } & \geqq p c^{\nu} \\
p\left(\underline{\alpha}^{\nu}+\underline{\beta}^{\nu}\right) & \leqq p \omega^{\nu} .
\end{aligned}
$$

The set of solutions of the reduced programme is denoted by $\mathcal{O}^{\nu}(p, w)$.

\section{UE exploitation: an axiomatic approach}

In the UE approach, exploitative relations are characterised by systematic differences between the labour that agents contribute to the economy and the labour 'received' by them, which is given by the amount of labour contained, or embodied, in some relevant consumption bundle(s). Therefore, in order to define exploitation status, it is necessary both to select the relevant bundle(s) and to identify their labour content. In economies with heterogeneous optimising agents and a general technology, neither choice is obvious, and various definitions have, in fact, been proposed.

The question, then, is which approach best captures the key insights of UE exploitation theory among those proposed, but also in the space of all conceivable definitions. In the literature, the proposal of alternative definitions has sometimes appeared as a painful process of adjustment of the theory to anomalies and counterexamples. In order to answer the question, and discriminate among a potentially infinite number of definitions, the axiomatic method pioneered by Yoshihara [41] seems more promising. An axiomatic approach suggests to start from first principles, thus explicitly identifying the class of suitable exploitation forms.

In his paper, Yoshihara [41] focuses on the Class-Exploitation Corresponding Principle (CECP; see Roemer [24]), which states that in equilibrium class membership and exploitation status emerge endogenously: the wealthy can rationally choose to belong to the capitalist class among other available options and become an exploiter, while the poor have no other option than being in the working class and are exploited. From this perspective, UE exploitative relations are relevant because they reflect unequal opportunities of life options, due to asset inequalities.

Under the classic definition by Okishio [20] and Morishima [19], CECP is proved as a formal theorem in simple Leontief production economies with rational agents (Roemer [24]; Veneziani [34]), but it does not hold in more 
general production economies (Roemer [24]; Yoshihara [41]). In contrast, Yoshihara [41] formulates CECP as an axiom capturing a key insight of UE exploitation theory on a generic feature of capitalist economy, and introduces a domain axiom that defines the class of admissible exploitation forms. Then, he derives a necessary and sufficient condition to identify the UE definitions that satisfy the domain axiom, and under which CECP holds in any general convex production economy (Yoshihara [41], Theorem 2). This condition allows us to test which UE definition within the appropriate domain preserves CECP in general. Interestingly, among the main definitions in the literature, an extension of the 'New Interpretation' form of exploitation (Duménil [5], [6]; Foley [11], [12]) is the only one that passes the test (Yoshihara [41], Corollaries 1-4).

In this paper, we focus more specifically on exploitation, rather than class. This section discusses a recent axiomatic analysis of UE exploitation theory based on Veneziani and Yoshihara [36]. An axiom called the ProfitExploitation Corresponding Principle (PECP), is presented which states that in equilibrium, the existence of positive profits corresponds to the social condition that every employed propertyless agent is exploited. This axiom is consistent with the traditional Marxian view that profits represent capitalist relations of production in which capitalists appropriate social surplus produced from the social labour of (propertyless) workers. But the nexus between profits, asset inequalities and the distribution of labour is relevant beyond Marxian theory. We then characterise the class of UE exploitationforms which satisfy PECP and a weak domain axiom.

In what follows, we focus on stationary RSs and examine UE exploitation and profits associated with the one-period allocations generated at a SRS. For the sake of notational simplicity, we denote SRSs simply by $(p, w)$ and any general convex economy as described in section 2 by $E$.

\subsection{The main definitions}

In this subsection, we introduce the main definitions of UE exploitation in the literature, suitably extended to economies with heterogeneous skills. Given any definition of exploitation, let $\mathcal{N}^{\text {ter }} \subseteq \mathcal{N}$ and $\mathcal{N}^{\text {ted }} \subseteq \mathcal{N}$ denote, respectively, the set of exploiters and the set of exploited agents at a given allocation, where $\mathcal{N}^{\text {ter }} \cap \mathcal{N}^{\text {ted }}=\varnothing$.

The classic and perhaps best known definition was provided by Okishio [20] in a simple Leontief economy, and was later generalised to the von Neu- 
mann economy by Morishima [19]. Formally, for all $c \in \mathbb{R}_{+}^{n}$, the minimum amount of (effective) labour necessary to produce $c$ as net output is:

$$
\text { l.v. }(c) \equiv \min \left\{\alpha_{l} \mid \alpha=\left(-\alpha_{l},-\underline{\alpha}, \bar{\alpha}\right) \in \phi(c)\right\} \text {. }
$$

By $\mathbf{A 0} \sim \mathbf{A} 2, \operatorname{l.v} .(c)$ is well-defined and is positive whenever $c \neq \mathbf{0}$ (Roemer, [23]). Then:

Definition 3 (Morishima [19]): Consider any $E \in \mathcal{E}$. For any $\nu \in \mathcal{N}$, who supplies $\Lambda^{\nu}$ and consumes $c^{\nu} \in \mathbb{R}_{+}^{n}: \nu \in \mathcal{N}^{\text {ted }}$ if and only if $\Lambda^{\nu}>$ l.v. $\left(c^{\nu}\right)$ and $\nu \in \mathcal{N}^{\text {ter }}$ if and only if $\Lambda^{\nu}<$ l.v. $\left(c^{\nu}\right)$.

Definition 3 is consistent with classical Marxian theory, in that UE exploitation is defined based upon the labour value of labour power, which is defined independently of price information. However, as argued by Roemer [24], a definition of exploitation independent of price information gives rise to counterintuitive results. Thus, a number of alternative definitions have been proposed, in which price information plays a crucial role.

Consider Roemer's ([24], chapter 5$)$ definition. Given a price vector $(p, w)$, the set of activities that yield the maximum profit rate is:

$$
P^{\pi}(p, w) \equiv\left\{\alpha \in \arg \max _{\alpha^{\prime} \in P} \frac{p \widehat{\alpha}^{\prime}-w \alpha_{l}^{\prime}}{p \underline{\alpha}^{\prime}}\right\}
$$

and the set of profit-rate-maximising activities that produce at least $c \in \mathbb{R}_{+}^{n}$ as net output is:

$$
\phi(c ; p, w) \equiv\left\{\alpha \in P^{\pi}(p, w) \mid \widehat{\alpha} \geqq c\right\} .
$$

For all $c \in \mathbb{R}_{+}^{n}$, the minimum amount of (effective) labour necessary to produce $c$ as net output among profit-rate-maximising activities is:

$$
\text { l.v. }(c ; p, w) \equiv \min \left\{\alpha_{l} \mid \alpha=\left(-\alpha_{l},-\underline{\alpha}, \bar{\alpha}\right) \in \phi(c ; p, w)\right\} .
$$

Again, l.v. $(c ; p, w)$ is well defined at SRSs and is positive for all $c \neq \mathbf{0}$. Then:

Definition 4 (Roemer [24]): Consider any $E \in \mathcal{E}$. Let $(p, w)$ be a SRS for $E$. For any $\nu \in \mathcal{N}$, who supplies $\Lambda^{\nu}$ and consumes $c^{\nu}: \nu \in \mathcal{N}^{\text {ted }}$ if and only if $\Lambda^{\nu}>$ l.v. $\left(c^{\nu} ; p, w\right)$ and $\nu \in \mathcal{N}^{\text {ter }}$ if and only if $\Lambda^{\nu}<$ l.v. $\left(c^{\nu} ; p, w\right)$. 
Finally, we analyse a definition recently proposed by Yoshihara and Veneziani [42], [43] and Yoshihara [41]. For any $p \in \mathbb{R}_{+}^{n}$ and $c \in \mathbb{R}_{+}^{n}$, let $\mathcal{B}(p, c) \equiv$ $\left\{x \in \mathbb{R}_{+}^{n} \mid p x=p c\right\}$ be the set of bundles that cost exactly as much as $c$ at prices $p$. Let $\alpha^{p, w} \equiv \sum_{\nu=1}^{N}\left(\alpha^{\nu}+\beta^{\nu}\right)$ denote the aggregate equilibrium production activity at a $\operatorname{SRS}(p, w)$ for $E$.

Definition 5 : Consider any $E \in \mathcal{E}$. Let $(p, w)$ be a $S R S$ for $E$ with aggregate production activity $\alpha^{p, w}$. For all $c \in \mathbb{R}_{+}^{n}$ with $p c \leqq p \widehat{\alpha}^{p, w}$, let $\tau^{c} \in[0,1]$ be such that $\tau^{c} \widehat{\alpha}^{p, w} \in \mathcal{B}(p, c)$. The labour embodied in $c$ at $\alpha^{p, w}$ is $\tau^{c} \alpha_{l}^{p, w}$.

As in Roemer's [24] approach, in Definition 5 the labour content of a bundle can be identified only if the price vector is known. Yet social relations play a more central role, because the definition of labour content requires a prior knowledge of the social reproduction point, and labour content is explicitly linked to the redistribution of total social labour, which corresponds to the total labour content of national income. Then:

Definition 6 : Consider any $E \in \mathcal{E}$. Let $(p, w)$ be a SRS for $E$ with aggregate production activity $\alpha^{p, w}$. For any $\nu \in \mathcal{N}$, who supplies $\Lambda^{\nu}$ and consumes $c^{\nu}$, let $\tau^{c^{\nu}}$ be defined as in Definition 5. Then $\nu \in \mathcal{N}^{\text {ted }}$ if and only if $\Lambda^{\nu}>\tau^{c^{\nu}} \alpha_{l}^{p, w}$ and $\nu \in \mathcal{N}^{\text {ter }}$ if and only if $\Lambda^{\nu}<\tau^{c^{\nu}} \alpha_{l}^{p, w}$.

Definition 6 is conceptually related to the 'New Interpretation' (NI) developed by Duménil [5], [6] and Foley [11], [12]: for all $\nu \in \mathcal{N}, \tau^{c^{\nu}}$ represents $\nu$ 's share of national income, and so $\tau^{c^{\nu}} \alpha_{l}^{p, w}$ is the share of social labour that $\nu$ receives by earning income barely sufficient to buy $c^{\nu}$. Then, as in the NI, the notion of exploitation is related to the production and distribution of national income and social labour.

\subsection{Labour exploitation}

In this section, a general domain condition is presented which captures the core insights of UE exploitation theory shared by all of the main approaches.

Let $\mathcal{W} \equiv\left\{\nu \in \mathcal{N} \mid \omega^{\nu}=\mathbf{0}\right\}$. The set $\mathcal{W}$ is of focal interest in exploitation theory: if any agents are exploited, then those with no initial endowments should be among them, if they work at all. It is therefore opportune, from an axiomatic viewpoint, to focus on $\mathcal{W}$ in order to identify some minimum requirements that all UE definitions should satisfy. 
Let $B(p, w \Lambda) \equiv\left\{c \in \mathbb{R}_{+}^{n} \mid p c=w \Lambda\right\}$ be the set of consumption bundles that can be just afforded, at prices $p$, by an agent in $\mathcal{W}$, who supplies $\Lambda$ units of labour at a wage $w$. We can now introduce the domain axiom.

Axiom 1 Labour Exploitation (LE): Consider any $E \in \mathcal{E}$. Let $(p, w)$ be a SRS for E. Given any definition of exploitation, the set $\mathcal{N}^{\text {ted }} \subseteq \mathcal{N}$ should have the following property at $(p, w)$ : there exists a profile $\left(c_{e}^{1}, \ldots, c_{e}^{|W|}\right)$ such that for any $\nu \in \mathcal{W}, c_{e}^{\nu} \in B\left(p, w \Lambda^{\nu}\right)$ and for some $\alpha^{c_{e}^{\nu}} \in \phi\left(c_{e}^{\nu}\right) \cap \partial P$ with $\widehat{\alpha}^{c_{e}^{\nu}} \ngtr c_{e}^{\nu}$ :

$$
\nu \in \mathcal{N}^{t e d} \Leftrightarrow \alpha_{l}^{c_{e}^{\nu}}<\Lambda^{\nu}
$$

LE requires that, at any SRS, the exploitation status of each propertyless worker $\nu \in \mathcal{W}$ be characterised by identifying a nonnegative vector $c_{e}^{\nu}$, that may be defined an exploitation reference bundle (ERB). The ERB must be technically feasible and on $\nu$ 's budget line, and it identifies the amount of labour that $\nu$ receives, $\alpha_{l}^{c_{e}^{\nu}}$. Thus, if $\nu \in \mathcal{W}$ supplies $\Lambda^{\nu}$, and $\Lambda^{\nu}$ is more than $\alpha_{l}^{c_{e}^{\nu}}$, then $\nu$ is regarded as contributing more labour than $\nu$ receives. According to $\mathbf{L E}$, all such agents belong to $\mathcal{N}^{\text {ted }}$.

In UE theory, the exploitation status of agent $\nu$ is determined by the difference between the amount of labour that $\nu$ 'contributes' to the economy, and the amount she 'receives'. As a domain condition for the admissible class of exploitation-forms, LE provides some minimal, key restrictions on the definition of the amount of labour that a theoretically relevant subset of agents contributes and the amount they receive.

According to LE, the former quantity is given by the effective labour, $\Lambda^{\nu}$, rather than the labour time, $\lambda^{\nu}$, performed by the agent. This is because, as a domain condition for UE exploitation, LE aims to capture the key intuitions common to all of the main approaches: not only is a focus on effective labour the natural extension of all of the classic definitions in the Okishio-Morishima-Roemer tradition, it is also the standard approach in the literature on exploitation in economies with heterogeneous labour and skills (see, e.g., Krause [14]; Duménil, Foley, and Lévy [7]). ${ }^{3}$ Moreover, by focusing on $\Lambda^{\nu}$, LE incorporates the key normative intuition of what may be called the 'contribution view' of exploitation theory: a UE exploitation-free allocation coincides with the proportional solution, a well-known fair allocation rule whereby every agent's income is proportional to her contribution to the

\footnotetext{
${ }^{3}$ For a slightly different, but related approach based on the notion of 'abstract labour', see Fleurbaey ([9], section 8.5).
} 
economy (Roemer and Silvestre [30]). Proportionality is a strongly justified normative principle, whose philosophical foundations can be traced back to Aristotle (Maniquet [15]) and which can be justified in terms of the Kantian categorical imperative (Roemer [29]).

LE imposes even weaker restrictions on the amount of labour received by the agents in $\mathcal{W}$. First, the amount of labour that $\nu \in \mathcal{W}$ receives depends on her income, or more precisely, it is determined in equilibrium by some reference bundle that $\nu$ can purchase. In the standard approaches, the ERB corresponds to the bundle actually chosen by the agent. In Definitions 3 and 4 , for example, $c_{e}^{\nu} \equiv c^{\nu} \in B\left(p, w \Lambda^{\nu}\right)$. Indeed, as noted by an anonymous referee, it may be argued that $\mathbf{L E}$ should explicitly require that $c_{e}^{\nu}=c^{\nu}$, for UE exploitation status should be defined based only on the information emerging from the actual exchange process. But this subjectivist view is not uncontroversial. Following the standard Marxian approach, for example, one may insist that exploitation status depend on productive decisions, and not on possibly arbitrary consumption decisions. From this viewpoint, agents who are identical in all characteristics except their consumption choices should have the same exploitation status.

At any rate, we need not adjudicate this issue. For our aim is to provide a weak domain condition that is shared by all of the main approaches. Therefore, LE does not rule out the possibility that $c_{e}^{\nu}=c^{\nu}$, but it does not impose it as a requirement and it only requires that the ERB be potentially affordable. Thus, in Definition 6 , given any $(p, w)$ with aggregate production activity $\alpha^{p, w}, c_{e}^{\nu} \equiv \tau^{c^{\nu}} \cdot \widehat{\alpha}^{p, w} \in B\left(p, w \Lambda^{\nu}\right)$, where $\tau^{c^{\nu}}=\frac{p c^{\nu}}{p \widehat{\alpha}^{p, w}}$.

Second, the amount of labour associated with the ERB - and thus 'received' by an agent - is related to production conditions: LE states that the ERB be technologically feasible as net output, and its labour content is the amount of labour socially necessary to produce it. Observe that LE requires that the amount of labour associated with each ERB be uniquely determined with reference to production conditions, but it does not specify how such amount should be chosen, and there may be many (efficient) ways of producing $c_{e}^{\nu}$, and thus of determining $\alpha_{l}^{c_{e}^{\nu}}$. In Definition $3, \alpha^{c_{e}^{\nu}} \in$ $\arg \min \left\{\alpha_{l} \mid \alpha \in \phi\left(c_{e}^{\nu}\right)\right\} ;$ in Definition $4, \alpha^{c_{e}^{\nu}} \in \arg \min \left\{\alpha_{l} \mid \alpha \in \phi\left(c_{e}^{\nu} ; p, w\right)\right\}$; and in Definition $6, \alpha^{c_{e}^{\nu}} \equiv \tau^{c^{\nu}} \alpha^{p, w}$, where $\tau^{c^{\nu}}=\frac{p c^{\nu}}{p \widehat{\alpha}^{p, w}}$.

Finally, note that $\mathbf{L E}$ does not provide comprehensive conditions for the determination of exploitation status: it only focuses on a subset of agents 
and it imposes no restrictions on the set of exploiters $\mathcal{N}^{\text {ter }} .{ }^{4}$

In summary, LE represents an appropriate domain condition in exploitation theory: it is formally weak and incorporates some widely shared views on UE exploitation. Thus, although it is not trivial and not all definitions in the literature satisfy it, all of the major approaches do. ${ }^{5}$ The next question, then, is how to discriminate among the various definitions satisfying $\mathbf{L E}$.

\subsection{The Profit-Exploitation Correspondence Principle}

A key tenet of UE exploitation theory is the idea that profits are one of the main determinants of the existence of exploitation, and of inequalities in well-being freedom: profits represent the way in which capitalists appropriate social surplus and social labour. Therefore a general correspondence should exist between positive profits and the exploitation of at least the poorest segments of the working class. This is formalised in the next axiom.

Axiom 2 Profit-Exploitation Correspondence Principle (PECP): For any $E \in \mathcal{E}$ and any $S R S$ for $E,(p, w)$, with aggregate production activity $\alpha^{p, w}$ :

$$
\left[p \widehat{\alpha}^{p, w}-w \alpha_{l}^{p, w}>0 \Leftrightarrow \mathcal{N}^{\text {ted }} \supseteq \mathcal{W}_{+}\right],
$$

whenever $\mathcal{W}_{+} \equiv\left\{\nu \in \mathcal{W} \mid \Lambda^{\nu}>0\right\} \neq \varnothing$.

Observe that PECP is formulated without specifying any definition of exploitation: whatever the definition adopted, propertyless agents should be exploited if and only if profits are positive in equilibrium. The axiom is weak in that it only focuses on a subset of $\mathcal{N}$ and it is silent on the set of exploiters $\mathcal{N}^{\text {ter }}$. Further, PECP is fairly general, because it both applies to economies with a complex class structure, and allows for the possibility that propertyless workers in $\mathcal{W}_{+}$are a strict subset of $\mathcal{N}^{\text {ted }}$. Note that the axiom focuses only on propertyless workers who perform some labour: this is theoretically appropriate, since the exploitation status of agents who do not engage in any economic activities is unclear. Finally, PECP allows for

\footnotetext{
${ }^{4}$ It is worth noting in passing that the vector $c_{e}^{\nu}$ in $\mathbf{L E}$ may be a function of $(p, w)$ and that once $c_{e}^{\nu}$ is identified, the existence of $\alpha^{c_{e}^{\nu}}$ is guaranteed by $\mathbf{A} \mathbf{2}$ and $\mathbf{A} \mathbf{3}$.

${ }^{5}$ Based on Flaschel's [8] notion of actual labour values, another definition can be derived which satisfies LE. Instead, the subjectivist notion of labour exploitation based on workers' preferences proposed by Matsuo [16] does not satisfy LE.
} 
fairly general assumptions on agents and technology, including heterogeneous preferences and skills, a convex technology, and so on.

It may be objected that PECP should not be considered as a postulate. In mathematical Marxian economics, and in Marx's own work, the equivalence between positive profits and the existence of (aggregate) exploitation has been traditionally derived as a theoretical result, as in the literature on the Fundamental Marxian Theorem (FMT; see Okishio [20] and Morishima [19]). As such, the link between exploitation and profits should hold under some conditions but not others, which seems prima facie inconsistent with the logical status of a postulate. ${ }^{6}$

This objection is not entirely compelling. Although the axiomatic approach has not been used explicitly in mathematical Marxian economics, the FMT has been de facto, albeit implicitly, considered as a key axiom in exploitation theory (and the same holds for the CECP). The central relevance of the FMT is suggested by its very name and it has been widely considered as "the core of [Marx's] economic theory" (Morishima [19]: 622) such that alternative definitions have been proposed and compared in the literature based on whether they preserved it (and the CECP). Roemer's interpretation of the CECP can indeed be extended to the FMT: although it is formally proved as a theorem, it defines the core of Marx's theory and thus "its epistemological status is as a postulate. We seek to construct models that allow us to prove it" (Roemer, [25]: 270).

To consider PECP as a postulate is therefore consistent with the central theoretical role assigned to the relation between exploitation and profits in the literature. Indeed, if an impossibility result followed from the imposition of PECP, this would arguably raise serious questions about some of the key intuitions of UE exploitation theory. And this is particularly relevant given that the PECP is significantly weaker than the FMT in that it imposes no constraints in equilibria where $\mathcal{W}_{+}=\varnothing$ and when equilibrium profits are zero it only requires that some propertyless agents not be exploited.

Theorem 7, however, characterises the non-empty class of exploitationforms that satisfy LE and such that PECP holds.

Theorem 7 (Veneziani and Yoshihara [36]) For any definition of labour exploitation satisfying $\boldsymbol{L} \boldsymbol{E}$, the following statements are equivalent for any $E \in \mathcal{E}$ and for any $S R S(p, w)$ with aggregate production activity $\alpha^{p, w}:$

\footnotetext{
${ }^{6}$ We are grateful to two anonymous referees for bringing this issue to our attention.
} 
(1) $\boldsymbol{P E C P}$ holds under this definition;

(2) if $\pi^{\max }>0$, then for each $\nu \in \mathcal{W}_{+}$, there exists $\alpha_{\pi}^{\nu} \in P\left(\alpha_{l}=\Lambda^{\nu}\right) \cap$ $\partial P$ such that $\widehat{\alpha}_{\pi}^{\nu} \in \mathbb{R}_{+}^{n}, p \widehat{\alpha}_{\pi}^{\nu}>w \Lambda^{\nu}$, and $\left(\alpha_{\pi l}^{\nu}, \underline{\alpha}_{\pi}^{\nu}, \bar{\alpha}_{\pi}^{\nu}\right) \geqq \eta^{\nu}\left(\alpha_{l}^{c_{e}^{\nu}}, \underline{\alpha}^{c_{e}^{\nu}}, \bar{\alpha}^{c_{e}^{\nu}}\right)$ for some $\eta^{\nu}>1$.

Theorem 7 can be interpreted as follows. PECP states that propertyless workers are exploited if and only if equilibrium profits are positive. According to $\mathbf{L E}$, the exploitation status of propertyless agents is determined by identifying a profile of (affordable) reference bundles which must be producible with less than $\Lambda^{\nu}$ units of labour for all exploited workers. By Theorem 7 , in every convex economy, PECP holds if and only if the existence of positive profits in equilibrium is also determined by identifying a profile of reference bundles $\left(\widehat{\alpha}_{\pi}^{\nu}\right)_{\nu \in \mathcal{W}_{+}}$. According to condition (2), for all $\nu \in \mathcal{W}_{+}$, these reference bundles must be producible with a technically efficient process using $\Lambda^{\nu}$ units of labour, and must be such that they are not affordable by $\nu$ and dominate the ERBs if the maximum profit rate is positive.

Theorem 7 does not identify a unique definition that meets PECP, but rather a class of definitions satisfying condition (2). Yet Veneziani and Yoshihara ([36], Corollary 1) show that it has surprising implications concerning the main approaches in exploitation theory. For there are economies in which for all $\nu \in \mathcal{W}_{+}$, condition (2) is never satisfied, if $\alpha^{c_{e}^{\nu}}$ is given by Definitions 3 or 4 and so the PECP does not hold. In contrast, Definition 6 satisfies condition (2), and thus PECP holds for all $E \in \mathcal{E}$ and all $\operatorname{SRS}(p, w)$.

Methodologically, Theorem 7 suggests that an axiomatic analysis provides interesting insights and has relevant implications. This conclusion is far from trivial: as noted by an anonymous referee, one may doubt that the definition of exploitation requires an axiomatic analysis and argue that it would be more interesting to use axioms to justify a measure of the degree of exploitation. Yet, as shown above, as soon as the simplest polarised, two-class economies with restrictive assumptions on preferences and technology are abandoned, different definitions of UE exploitation have very different properties and incorporate different normative and positive insights. An axiomatic analysis of the definition of exploitation is therefore useful, if not necessary, in order to adjudicate the possible alternative views, before one can actually tackle the issue of the degree of exploitation.

Theorem 7 provides a demarcation line (condition 2) by which one can test which of infinitely many potential definitions preserves the relation between exploitation and profits in capitalist economies. Indeed, it characterises the 
class of definitions which are coherent, in the sense of preserving such relation regardless of the complexity of economic models. Note that Theorem 7 immediately implies that in simple Leontief economies with homogeneous agents, any definition of UE exploitation within the domain given by LE satisfies PECP. Yet in more complex economies, many of the definitions proposed in the literature violate PECP. Rather than concluding that there exists no general relation between exploitation and profits in capitalist economies, it seems more apt to consider these definitions as incoherent, or at least as not being robust. For both the Leontief model and the more general economies analysed in this paper represent competitive market economies with differential ownership of productive assets, and the differences between the two do not reflect different stages of development of capitalist societies, or differences in degrees of income disparity and social productivity, or in uneven power relations in the capitalist production process. Hence, there is no reason why the basic implications of exploitative social relations should vary according to the technical complexity of the economic models.

Substantively, the above arguments, and other recent axiomatic analyses, provide significant support to Definition 6, as the appropriate definition of UE exploitation. Theorem 7 proves that, unlike the main competing definitions, the NI preserves one of the key insights of classic exploitation theory.

Actually, not only does Theorem 7 establish that the set of definitions that preserve the PECP is not empty: if Definition 6 is adopted, the existence of profits is synonymous with the exploitation of labour. A traditional objection moved against the FMT in the Okishio-Morishima-Roemer approach is that the existence of profits is equivalent to the productiveness of the economy, which in turn is equivalent to the 'exploitation' of any commodity (see the Generalised Commodity Exploitation Theorem, Roemer [24]), which raises doubts on the significance of the FMT. Yoshihara and Veneziani [44] have proved that the NI captures exploitation as the unequal exchange of labour: unlike all other approaches, the existence of UE exploitation is not synonymous with the existence of any commodity exploitation.

Indeed, the NI may provide the foundations for a general theoretical framework that can deal with many unresolved issues in exploitation theory. Definition 6 can be easily extended to the general economies described in section 2 and, as Veneziani and Yoshihara ([36], Theorem 2) have shown, it is possible to determine the exploitation status of all agents and the whole exploitation structure of such general economies in equilibrium. Moreover, a robust relationship between profits and exploitation can be proved even at 
disequilibrium allocations (Veneziani and Yoshihara [36], Theorem 3).

Definition 6 has a clear empirical content, for it is firmly anchored to the actual data of the economy and, unlike Definitions 3 and 4, it does not require information about all conceivable production techniques: only actual production decisions and the social allocation of labour, income and production activities matter. Indeed, as Yoshihara and Veneziani [43] have shown, it also satisfies a property of Minimal objectivism in that it does not rely on information about agents' subjective preferences and possibly arbitrary consumption decisions.

Perhaps more importantly, from a normative perspective, Definition 6 conceptualises exploitation as a social relation. Not only is the notion of exploitation related to the production and distribution of national income and social labour, as noted above. It can be proved that, unlike the main definitions in the literature, the NI identifies the existence of exploitative relations, in that some agents are exploited if and only if there is someone exploiting them (Yoshihara and Veneziani [42], Veneziani and Yoshihara [36]).

Finally, Definition 6 identifies exploitative relations as characterised by inequalities in individual income/labour ratios - an important normative intuition of the UE approach which provides an interesting conceptual link with liberal egalitarian approaches.

\section{The dynamics of exploitation}

The previous section provides an axiomatic analysis of the distributive aspects of UE exploitation. Yet, a fundamental and contentious question in exploitation theory concerns precisely the role of distributive issues, on the one hand, and of relations of coercion, force, or power, on the other hand. At the most general level, $A$ exploits $B$ if and only if $A$ takes unfair advantage of $B$. But do exploitative relations mainly, or uniquely, involve some (wrongful) characteristic of the structure of the interaction between $A$ and $B$ (such as asymmetric relations of power, force, coercion, etc.)? Or is exploitation mainly, or uniquely, concerned with some form of (wrongful) inequality (in asset ownership, labour exchanged, income, etc.)?

A path-breaking answer to these questions is provided by John Roemer's seminal theory [24], [26]. Roemer's key conclusion is that all relevant moral information is conveyed by the analysis of Differential Ownership of Productive Assets (henceforth, DOPA) and the resulting welfare inequalities. 
Notions of power or dominance are not relevant. On the one hand, Roemer rejects all approaches based on domination at the point of production or coercion in the labour market. As Roemer put it, 'Capitalism's necessary coercions are economic: ... it can substantially rid itself ... of extra-economic coercions, such as domination in the workplace ... Such a capitalism might be kinder and gentler, as they say, but it would not be socialism' (Roemer [27]: 386). On the other hand, he proves that the labour market is not 'intrinsically necessary for bringing about the Marxian phenomena of exploitation and class ... competitive markets and [DOPA] are the institutional culprits in producing exploitation and class' (Roemer [24]: 93).

Consequently, Roemer has developed an alternative game theoretic approach that focuses on property relations, which aims to generalise Marxian exploitation 'in terms of the institutional variation permitted' (Roemer [25]: 256) and to capture its essential normative content, which is interpreted as requiring an egalitarian distribution of resources in the external world.

Roemer is effective in criticising approaches that focus on domination and direct coercion, and in stressing the relevance of distributive issues. It is however unclear that weaker forms of asymmetric relations between agents can, or indeed should be ruled out. Concerning Roemer's philosophical argument, Veneziani [35] has shown that purely distributive approaches to exploitation have too impoverished an informational basis to capture exploitative relations and to distinguish exploitation from other forms of injustice, or wrongs. Roemer's own game theoretic approach somewhat paradoxically casts doubts on the idea that relations of power should be ruled out. ${ }^{7}$

Perhaps more importantly, it is unclear that Roemer's formal argument convincingly establishes that exploitation can be reduced to a focus on DOPA. For 'The economic problem for Marx, in examining capitalism, was to explain the persistent accumulation of wealth by one class and the persistent impoverishment of another, in a system characterized by voluntary trade' (Roemer [24]: 6, italics added). Roemer's models, however, are essentially static in that there are no intertemporal trade-offs, and so they are not suitable for analysing the persistence of exploitation in a capitalist economy.

In this section, based on the general model set up in section 2 above, we survey and extend some recent contributions that analyse exploitation in an intertemporal context (Veneziani [34], [35]). A dynamic generalisation of

\footnotetext{
${ }^{7}$ In later writings, Roemer himself has acknowledged the limits of purely distributive definitions. See, for example, Roemer [27] and, for a discussion, Veneziani [35].
} 
Roemer's [24] subsistence economies is analysed in order to assess the relevance of DOPA, focusing on its role in generating exploitation as a persistent feature of a competitive economy with savings and a variable distribution of productive assets. We analyse subsistence economies because this allows us to examine the role of DOPA in a context where capital scarcity persists. In fact, the results obtained in Roemer's static economies depend on differential ownership of scarce productive assets (Skillman [31]) and it is not too surprising that exploitation may disappear when accumulation is allowed (Devine and Dymski [4]). Moreover, Roemer's main conclusions do not depend on accumulation. On the contrary, one of his key results is precisely that 'exploitation emerges logically prior to accumulation' (Roemer [25]: 264).

\subsection{Subsistence economies: equilibrium}

A subsistence economy is a special case of the economies analysed in section 2 in which agents are endowed with identical preferences and skills, and wish to minimise labour time subject to earning enough to purchase a given subsistence bundle. Formally, a convex economy $E\left(P, \mathcal{N}, C,\left(u^{\nu}\right)_{\nu \in \mathcal{N}},\left(\varsigma^{\nu}\right)_{\nu \in \mathcal{N}}, \Omega_{k T}\right)$ is a subsistence economy if for all $\nu \in \mathcal{N}$ : (i) there exists a bundle $b \in$ $\mathbb{R}_{+}^{n} \backslash\{\mathbf{0}\}$ which must be consumed in order to survive in each period, so that $C=C_{b} \equiv\left\{c \in \mathbb{R}_{+}^{n} \mid c \geqq b\right\}$; (ii) $u^{\nu}(c, \lambda)=u_{b}(c, \lambda) \equiv 1-\lambda$, for all $(c, \lambda) \in C_{b} \times[0,1]$, and (iii) $\varsigma^{\nu}=1$. Denote a subsistence economy by a list $E\left(P, \mathcal{N}, C_{b}, u_{b}, 1, \Omega_{k T}\right)$ or, as a shorthand notation, $E_{b}\left(\Omega_{k T}\right)$.

In any $E_{b}\left(\Omega_{k T}\right)$, the individual optimisation programme $M P^{\nu}$ is a special case of the general programme in section 2.2, where the objective function is $\sum_{t=k T}^{(k+1) T-1}-\rho^{t} \Lambda_{t}^{\nu}$ and in each period $c_{t}^{\nu}=b$, without loss of generality. Accordingly, Definition 1 is slightly revised.

Definition 8 A reproducible solution (RS) for $E_{b}\left(\Omega_{k T}\right)$ is a price vector $(\mathbf{p}, \mathbf{w})$ and an associated set of actions such that:

(i) $\xi^{\nu} \in \mathcal{O}^{\nu}(\mathbf{p}, \mathbf{w})$, all $\nu$;

(ii) $\widehat{\alpha}_{t}+\widehat{\beta}_{t} \geqq N b+s_{t}$, all $t$;

(iii) $\underline{\alpha}_{t}+\underline{\beta}_{t}+\delta_{t} \leqq \omega_{t}$, all $t$;

(iv) $\beta_{l t}=\bar{\gamma}_{t}$, all $t$;

(v) $\omega_{(k+1) T} \geqq \omega_{k T}$.

In what follows, unless otherwise stated, only non-trivial RS's are considered in which some production takes place in every period. Given any 
$(\mathbf{p}, \mathbf{w})$, let $\pi_{t}^{\max } \equiv \max _{\alpha \in P} \frac{p_{t} \bar{\alpha}-p_{t-1} \underline{\alpha}-w_{t} \alpha_{l}}{p_{t-1} \underline{\alpha}}$ : at a non-trivial RS, it must be $\pi_{t}^{\max } \geqq \max _{i} \frac{p_{i t}-p_{i t-1}}{p_{i t-1}}$, all $t$. For if $\pi_{t}^{\max }<\max _{i} \frac{p_{i t}-p_{i t-1}}{p_{i t-1}}$, then at the solution to $M P^{\nu}, \alpha_{t}^{\nu}+\beta_{t}^{\nu}=\mathbf{0}$ holds for all $\nu \in \mathcal{N}$. Therefore, for all $\nu \in \mathcal{N}$ who work in equilibrium, we can set $\delta_{t}^{\nu}=0$ without loss of generality.

In order to avoid uninteresting technicalities, following Roemer [24], [26] we assume that agents who can reproduce themselves without working use the amount of wealth strictly necessary to obtain their subsistence bundle $b$.

Non Benevolent Capitalists (NBC): If agent $\nu$ has a solution to $M P^{\nu}$ with $\Lambda_{t}^{\nu}=0$, all $t$, then $\nu$ chooses $\xi^{\nu}$ to satisfy $\left(1+\pi^{\max }\right) p_{t-1}\left(\underline{\alpha}_{t}^{\nu}+\underline{\beta}_{t}^{\nu}\right)=$ $p_{t} b+p_{t} \omega_{t+1}^{\nu}$ at each $t$.

Lemma 9 states that at a $\mathrm{RS}$, at all $t$, the revenues constraint binds for all agents and, if the equilibrium profit rate is positive, the wealth constraint binds, for all $\nu$ who work.

Lemma 9 Let $(\mathbf{p}, \mathbf{w})$ be a $R S$ for $E_{b}\left(\Omega_{k T}\right)$. Then:

(i) under $\boldsymbol{N B C},\left[p_{t} \bar{\alpha}_{t}^{\nu}\right]+\left[p_{t} \bar{\beta}_{t}^{\nu}-w_{t} \beta_{l t}^{\nu}\right]+w_{t} \gamma_{t}^{\nu}=p_{t} b+p_{t} \omega_{t+1}^{\nu}$, all $t, \nu$;

(ii) if $\pi_{t}^{\max }>0$ all $t$, and $\sum_{t=k T}^{(k+1) T-1} \Lambda_{t}^{\nu}>0$ all $\xi^{\nu} \in \mathcal{O}^{\nu}(\mathbf{p}, \mathbf{w})$, then $p_{t-1}\left(\underline{\alpha}_{t}^{\nu}+\underline{\beta}_{t}^{\nu}\right)=p_{t-1} \omega_{t}^{\nu}$, all $t$.

Lemma 10 derives some properties of equilibrium prices in every $t$.

Lemma 10 Let $(\mathbf{p}, \mathbf{w})$ be a $R S$ for $E_{b}\left(\Omega_{k T}\right)$. Then, for all $t$, (i) $p_{t} \bar{\alpha}-p_{t-1} \underline{\alpha}-$ $w_{t} \alpha_{l} \geqq 0$, for some $\alpha \in P \backslash\{\mathbf{0}\}$; (ii) $p_{t} \geq \mathbf{0}$ with $p_{t} b>0$; and (iii) $w_{t}>0$.

The proofs of Lemmas 9 and 10 are straightforward and therefore omitted. Proposition 11 derives labour expended by each agent in each period.

Proposition 11 Let $(\mathbf{p}, \mathbf{w})$ be a $R S$ for $E_{b}\left(\Omega_{k T}\right)$. Then, for all $t, \nu: \Lambda_{t}^{\nu}=$ $\max \left\{0, \frac{p_{t} b-\left(1+\pi_{t}^{\max }\right) p_{t-1} \omega_{t}^{* \nu}+p_{t} \omega_{t+1}^{* \nu}}{w_{t}}\right\}$.

Proof. If $\sum_{t=k T}^{(k+1) T-1} \Lambda_{t}^{\nu}>0$ for all $\xi^{\nu} \in \mathcal{O}^{\nu}(\mathbf{p}, \mathbf{w})$, the result immediately follows from Lemma 9. If there is a $\xi^{\nu}$ such that $\sum_{t=k T}^{(k+1) T-1} \Lambda_{t}^{\nu}=0$, then $\Lambda_{t}^{\nu}=0$ and, by Lemma $9(\mathrm{i}), p_{t} b-\left(1+\pi_{t}^{\max }\right) p_{t-1} \omega_{t}^{* \nu}+p_{t} \omega_{t+1}^{* \nu} \leqq 0$ all $t$.

Proposition 12 describes a dynamic property of equilibrium prices. 
Proposition 12 Let $(\mathbf{p}, \mathbf{w})$ be a $R S$ for $E_{b}\left(\Omega_{k T}\right)$ such that at all $t$ there is some $\nu \in \mathcal{N}$ such that $p_{t-1} \omega_{t}^{\nu}>0$ and $\Lambda_{t}^{\nu} \in(0,1)$. Then, $\frac{1}{w_{t}}=\rho(1+$ $\left.\pi_{t+1}^{\max }\right) \frac{1}{w_{t+1}}$, all $t$.

Proof. By the convexity of $M P^{\nu}$, we can consider solutions with $\alpha_{t}^{\nu}=\mathbf{0}$, for all $t$ and all $\nu \in \mathcal{N}$ without loss of generality.

Take any $t$ and consider $\nu \in N$ such that $p_{t-1} \omega_{t}^{\nu}>0$ and $\Lambda_{t}^{\nu} \in(0,1)$. By Proposition 11

$$
\Lambda_{t}^{* \nu}=\gamma_{t}^{* \nu}=\frac{p_{t} b-\pi_{t}^{\max } p_{t-1} \underline{\beta}_{t}^{* \nu}+p_{t} s_{t}^{* \nu}+\left(p_{t}-p_{t-1}\right) \omega_{t}^{* \nu}}{w_{t}} .
$$

Then,

$$
\begin{aligned}
u_{t}^{\nu}+\rho u_{t+1}^{\nu}= & -\left[\Lambda_{t}^{* \nu}+\rho \Lambda_{t+1}^{* \nu}\right]=\frac{-p_{t} b+\pi_{t}^{\max } p_{t-1} \underline{\beta}_{t}^{* \nu}-p_{t} s_{t}^{* \nu}-\left(p_{t}-p_{t-1}\right) \omega_{t}^{* \nu}}{w_{t}}+ \\
& +\rho \frac{-p_{t+1} b+\pi_{t+1}^{\max } p_{t} \underline{\beta}_{t+1}^{* \nu}-p_{t+1} s_{t+1}^{* \nu}-\left(p_{t+1}-p_{t}\right) \omega_{t+1}^{* \nu}}{w_{t+1}} .
\end{aligned}
$$

Consider a one-period perturbation $s_{t}^{\prime \nu}=s_{t}^{* \nu}+\Delta_{t}^{\nu}, s_{t+1}^{\prime \nu}=s_{t+1}^{* \nu}+\Delta_{t+1}^{\nu}$, such that $\Delta_{t}^{\nu}=-\Delta_{t+1}^{\nu}$. In the perturbed path,

$$
\begin{aligned}
u_{t}^{\prime \nu}+\rho u_{t+1}^{\prime \nu} & =-\left[\Lambda_{t}^{* \nu}+\rho \Lambda_{t+1}^{* \nu}\right]-\frac{p_{t} \Delta_{t}^{\nu}}{w_{t}}+\rho \frac{\pi_{t+1}^{\max } p_{t} \Delta_{t}^{\nu}-p_{t} \Delta_{t+1}^{\nu}}{w_{t+1}} \\
& =u_{t}^{\nu}+\rho u_{t+1}^{\nu}-\left[\frac{1}{w_{t}}-\rho \frac{\left(1+\pi_{t+1}^{\max }\right)}{w_{t+1}}\right] p_{t} \Delta_{t}^{\nu} .
\end{aligned}
$$

Note that at a non-trivial RS it must be $p_{i t}>0$, some $i$ for all $t$. Therefore, if $\frac{1}{w_{t}}<\rho \frac{\left(1+\pi_{t+1}^{\max }\right)}{w_{t+1}}$, then there is a sufficiently small $\Delta_{t}^{\nu} \geq \mathbf{0}$ that is feasible and generates $u_{t}^{\prime \nu}+\rho u_{t+1}^{\prime \nu}>u_{t}^{\nu}+\rho u_{t+1}^{\nu}$, contradicting optimality. A similar argument holds for $\frac{1}{w_{t}}<\rho \frac{\left(1+\pi_{t+1}^{\max }\right)}{w_{t+1}}$. Hence, $\frac{1}{w_{t}}=\rho \frac{\left(1+\pi_{t+1}^{\max }\right)}{w_{t+1}}$.

In what follows, RSs with stationary capital are of focal interest. As argued below, in equilibria with savings, some of the basic insights of Roemer's analysis do not hold. Moreover given the absence of population growth and technical progress, a RS with stationary capital implies that aggregate capital at the beginning of each generation's life is already optimal in terms of the 'golden rule'. For a sufficiently large $T$, if the initial aggregate capital stock 
was not at the optimal level, then agents would accumulate up to the optimal level as soon as possible and spend most of their lives with this optimal level of capital stock in order to minimise labour, as in the so-called Turnpike Theorem (see Morishima [17]). Therefore, we focus on RSs with stationary capital, and persistent capital scarcity, by assuming that aggregate capital is already at the optimal level in the initial period. Formally:

Definition 13 An interior reproducible solution (IRS) for $E_{b}\left(\Omega_{k T}\right)$ is a $R S$ $\left\langle(\mathbf{p}, \mathbf{w}),\left(\xi^{\nu}\right)_{\nu \in N}\right\rangle$ such that $s_{t}^{\nu}=\mathbf{0}$ for all $\nu \in \mathcal{N}$ at every $t$.

\subsection{Two views of UE exploitation in dynamic contexts}

In what follows, we abstract from unnecessary technicalities in our dynamic analysis and assume that there is only one consumption good, and the technology is of a simple Leontief type. Formally, the subsistence bundle is $b>0$, and the production set is $P=P_{(A, L)}$ for a Leontief technology $(A, L)$, with $A \in(0,1)$, and $L>0$. We adopt the standard notation: in every $t, x_{t}^{\nu}$ represents $\nu$ 's activity level as a self-employed producer, and $y_{t}^{\nu}$ is the activity level that $\nu$ hires others to operate. Thus, for any $\left(x_{t}^{\nu}, y_{t}^{\nu}, \gamma_{t}^{\nu}\right)$, there is $\left(\alpha_{t}^{\nu}, \beta_{t}^{\nu}, \gamma_{t}^{\nu}\right) \in P_{(A, L)} \times P_{(A, L)} \times[0,1]$ such that $\alpha_{t}^{\nu}=\left(-L x_{t}^{\nu},-A x_{t}^{\nu}, x_{t}^{\nu}\right)$, and $\beta_{t}^{\nu}=\left(-L y_{t}^{\nu},-A y_{t}^{\nu}, y_{t}^{\nu}\right)$, and a similar notation holds at the aggregate level. Hence, we use the notation $\left(x_{t}^{\nu}, y_{t}^{\nu}, \gamma_{t}^{\nu}\right)$ and $x_{t}+y_{t}$ to denote, respectively, individual plans and the aggregate production activity.

Let $v=L(1-A)^{-1}$. In one-good economies with a linear production technology, the labour content of a vector $c$ is simply $v c$ in all of the main approaches. Let $\Delta^{\nu}=\sum_{t=k T}^{(k+1) T-1}\left(\Lambda_{t}^{\nu}-v b\right)$. Unlike in the static model, there are two different criteria to define the exploitation status of an agent, focusing on the amount of labour performed either in each period, or during her whole life.

Definition 14 Agent $\nu$ is exploited within period $t$, or $W P_{t}$ exploited, iff $\Lambda_{t}^{\nu}>v b$; and a $W P_{t}$ exploiter iff $\Lambda_{t}^{\nu}<v b$. Similarly, $\nu$ is exploited during her whole life, or WL exploited, iff $\Delta^{\nu}>0$; and a WL exploiter iff $\Delta^{\nu}<0$.

The $W P$ and $W L$ definitions incorporate different normative concerns. The $W L$ definition captures the intuition that, from an individual's viewpoint, to be exploited in every period is certainly worse than being exploited only in some periods. This criterion may lead us to conclude that, from 
a perspective of individual well-being, an exploitative economy with social mobility is better than an exploitative economy without it.

Marx's idea, however, is more radical: the existence of exploitation is morally relevant per se, and exploitation may be considered as a property of the economy as a whole, not just of individuals. The $W P$ definition captures this intuition: the existence of $W P_{t}$ exploited agents and $W P_{t}$ exploiters implies the existence of exploitative social relations as a property of the whole economy. Further, an analysis based on the WL definition can only partly capture the exploitative structure of the economy, because it may lead to the conclusion that there would be no exploitation in 'changing places capitalism,' that is in a capitalist economy with significant social mobility, where WP exploitation exists in every period but the agents' status changes over time so as to equalise lifetime labour hours, which is rather counterintuitive from a Marxian perspective. Hence, although both criteria convey normatively relevant information, we focus mainly on the $W P$ definition, which is also more suitable to analyse the dynamics of exploitation.

If agents save, it may be difficult to extend Roemer's asset-based theory of exploitation to the dynamic context: given the optimality of $\sum_{t=k T}^{(k+1) T-1} s_{t}^{\nu}=$ 0 for all $\nu$, and the linearity of $M P^{\nu}$, an agent can be a $W P_{t}$ exploiter while being $W P_{t+j}$ exploited, for some $j \neq 0$, depending on the path of savings (and only indirectly on $\omega_{k T}^{\nu}$ ). Such changes in $W P$ status, however, do not necessarily convey morally relevant information: the fact that at a non-interior RS a relatively wealthy agent might optimally work more than $v b$ in $t$, in order to accumulate assets and minimise labour in $t+j$, does not raise serious moral concerns. Actually, it is not difficult to show that if $s_{t} \neq 0$ then there is no conceptual equivalence between $W P$ exploitative and inegalitarian solutions: only at an IRS, if an agent works less than $v b$, there must be another agent working more than $v b .^{8}$

Given our focus on IRSs, in what follows we consider $k T=0$ without loss of generality. By Lemma 10, at an IRS, we can set $p_{t}=p$, all $t$, and consider equilibrium price vectors of the form $(p, \mathbf{w})$. Finally, in the one-good economy, at any $t$ the profit rate is denoted more simply as $\pi_{t}$.

For any $(p, \mathbf{w})$, let $W_{t}^{*} \equiv\left(p b-w_{t} v b\right) / \pi_{t}$. Proposition 15 proves that at an IRS, the $W L$ and $W P$ definitions are equivalent and it extends Roemer's

\footnotetext{
${ }^{8}$ This argument does not apply to the $W L$ definition: the existence of a general monotonic relationship between initial wealth and $W L$ exploitation at a $\mathrm{RS}$ where agents save is an interesting issue for further research.
} 
asset-based theory of exploitation to the dynamic context.

Proposition 15 Let $(p, \mathbf{w})$ be an IRS for $E\left(\Omega_{0}\right)$ with $\pi_{0}>0$. Then:

1. $\Delta^{\nu}>0$ and $\Lambda_{t}^{\nu}>v b$, all $t$, if and only if $W_{0}^{\nu}<W_{0}^{*}$;

2. $\Delta^{\nu}=0$ and $\Lambda_{t}^{\nu}=v b$, all $t$, if and only if $W_{0}^{\nu}=W_{0}^{*}$;

3. $\Delta^{\nu}<0$ and $\Lambda_{t}^{\nu}<v b$, all $t$, if and only if $W_{0}^{\nu}>W_{0}^{*}$.

Proof. 1. At all $t, W_{t}^{\nu}=W_{t}^{*}$ is equivalent to $\pi_{t} W_{t}^{\nu}=\left[p(1-A)-w_{t} L\right](1-$ $A)^{-1} b$, or $p \omega_{0}^{\nu}=p A(1-A)^{-1} b$. Thus, if $W_{t}^{\nu}=W_{t}^{*}$, then $W_{t+1}^{\prime \nu}=W_{t+1}^{*}$, all $t$. Similarly, $W_{t}^{\nu}>W_{t}^{\mu}$ implies $W_{t+1}^{\nu}>W_{t+1}^{\mu}$ for any $\nu, \mu$, and all $t$.

2. By Proposition 11 and the strict monotonicity of $p\left[b-\pi_{t} \omega_{0}^{\nu}\right]$ in $W_{t}^{\nu}$ at all $t: \Lambda_{t}^{\nu}>v b \Leftrightarrow W_{t}^{\nu}<W_{t}^{*}, \Lambda_{t}^{\nu}=v b \Leftrightarrow W_{t}^{\nu}=W_{t}^{*}$, and $\Lambda_{t}^{\nu}<v b \Leftrightarrow$ $W_{t}^{\nu}>W_{t}^{*}$. Hence, by step $1, \Lambda_{0}^{\nu}>v b$ implies $\Lambda_{t}^{\nu}>v b$ all $t>0$, and thus $\Delta^{\nu}>0$. Conversely, if $\Delta^{\nu}>0$, it must be $\Lambda_{t}^{\nu}>v b$ for at least some $t \geqq 0$. However, as just shown, $W P$ exploitation status cannot change over time, and thus $\Lambda_{t}^{\nu}>v b$, all $t$. The other two cases are proved similarly.

\subsection{Exploitation, DOPA and welfare inequalities}

Given Proposition 15, it is natural to focus on IRSs in order to analyse the links between exploitation and wealth. The next results derive the conditions under which Roemer's [24],[26] theory can be extended to the intertemporal context, and at the same time highlight the conceptual links and differences between his definition of exploitation and neoclassical welfare inequalities.

Theorem 16 Let $\pi^{\prime}=(1-\rho) / \rho$ and let $\left(p, w^{\prime}\right)$ be the associated price vector. If $w_{t}=w^{\prime}$ all $t$, and $p b \leq w^{\prime}$, then for all $\nu, s_{t}^{\nu}=0$, all $t$, is optimal. Moreover, if $T$ is finite, then $V\left(\omega_{0}^{\nu}\right)=\max \left\{0,\left(1-\rho^{T}\right)\left[\frac{p^{\prime} b}{(1-\rho)}-\frac{p^{\prime} \omega_{0}^{\nu}}{\rho}\right]\right\}$, while if $T \rightarrow \infty$, then $V\left(\omega_{0}^{\nu}\right)=\max \left\{0, \frac{p^{\prime} b}{(1-\rho)}-\frac{p^{\prime} \omega_{0}^{\nu}}{\rho}\right\}$, where $p^{\prime} \equiv \frac{p}{w^{\prime}}$.

Proof. 1. Suppose $p \omega_{0}^{\nu} \geqq p b \rho /(1-\rho)$. The vector $\xi^{\nu}$ such that $s_{t}^{\nu}=0$ all $t$, and $y_{t}=y^{\prime}$ all $t$, with $\pi^{\prime} A y^{\prime}=b$, is optimal and $\Lambda_{t}^{\nu}=0$ all $t$.

Suppose $p \omega_{0}^{\nu}<p b \rho /(1-\rho)$, so that $\Lambda^{\nu}>0$ for all $\xi^{\nu} \in \mathcal{O}^{\nu}\left(p, w^{\prime}\right)$. Write $M P^{\nu}$ using dynamic optimisation theory. Let $\Psi: \mathbb{R}_{+} \rightarrow \mathbb{R}_{+}$be the feasibility correspondence:

$$
\Psi\left(\omega_{t}^{\nu}\right)=\left\{\omega_{t+1}^{\nu} \in \mathbb{R}_{+} \mid \frac{p}{w_{t}} \omega_{t+1}^{\nu} \leqq 1-\frac{p}{w_{t}} b+\frac{p}{w_{t}} \omega_{t}^{\nu}+\pi_{t} \frac{p}{w_{t}} \omega_{t}^{\nu}\right\} .
$$


Given $\omega_{0}^{\nu}$, let

$$
\Pi\left(\omega_{0}^{\nu}\right)=\left\{\omega^{\nu} \mid \omega_{t+1}^{\nu} \in \Psi\left(\omega_{t}^{\nu}\right) \text { all } t, \& \omega_{T}^{\nu} \geqq \omega_{0}^{\nu}\right\}
$$

be the set of feasible sequences $\omega^{\nu}$. Let $\Phi=\left\{\left(\omega_{t}^{\nu}, \omega_{t+1}^{\nu}\right) \in \mathbb{R}_{+} \times \mathbb{R}_{+} \mid \omega_{t+1}^{\nu} \in \Psi\left(\omega_{t}^{\nu}\right)\right\}$ be the graph of $\Psi$. The one-period return function $F: \Phi \rightarrow \mathbb{R}_{+}$at $t$ is $F\left(\omega_{t}^{\nu}, \omega_{t+1}^{\nu}\right)=\frac{p}{w_{t}} b+\frac{p}{w_{t}}\left(\omega_{t+1}^{\nu}-\omega_{t}^{\nu}\right)-\pi_{t} \frac{p}{w_{t}} \omega_{t}^{\nu} . M P^{\nu}$ becomes

$$
V\left(\omega_{0}^{\nu}\right)=\min _{\omega^{\nu} \in \Pi\left(\omega_{0}^{\nu}\right)} \sum_{t=0}^{T-1} \rho^{t}\left[\frac{p}{w_{t}} b+\frac{p}{w_{t}}\left(\omega_{t+1}^{\nu}-\omega_{t}^{\nu}\right)-\pi_{t} \frac{p}{w_{t}} \omega_{t}^{\nu}\right] .
$$

If $\frac{p b-\pi_{t} p \omega_{t}^{\nu}}{w_{t}} \leqq 1$ for all $t$, then $\Psi\left(\omega_{t}^{\nu}\right) \neq \varnothing$ for all $\omega_{t}^{\nu} \in \mathbb{R}_{+}$. Then, since $F$ is continuous and bounded, $M P^{\nu}$ is well defined for all $T$.

2. If $w_{t}=w^{\prime}$ for all $t$, then $\frac{p b-\pi_{t} p \omega_{t}^{\nu}}{w_{t}} \leqq 1$ for all $t, \nu$, and $M P^{\nu}$ becomes:

$$
V\left(\omega_{0}^{\nu}\right)=\min _{\omega^{\nu} \in \Pi\left(\omega_{0}^{\nu}\right)} \sum_{t=0}^{T-1} \rho^{t} p^{\prime} b+\rho^{T-1} p^{\prime} \omega_{T}^{\nu}-\left(1+\pi^{\prime}\right) p^{\prime} \omega_{0}^{\nu}, \text { where } p^{\prime} \equiv \frac{p}{w^{\prime}} .
$$

Therefore, for all $T$, any feasible $\omega^{\nu}$ such that $\omega_{T}^{\nu}=\omega_{0}^{\nu}\left(\right.$ or $\lim _{T \rightarrow \infty} \omega_{T}^{\nu}=$ $\omega_{0}^{\nu}$, if $\left.T \rightarrow \infty\right)$ is optimal and $V\left(\omega_{0}^{\nu}\right)$ immediately follows.

3. The last part of the statement is straightforward.

At an IRS, if $\pi_{t}=\pi^{\prime}=(1-\rho) / \rho$, all $t$, then $\left(p, w_{t}\right)=\left(p, w^{\prime}\right)$, all $t$, and so the IRS is a stationary $R S$ (SRS).

Given Theorem 16, the next result characterises welfare inequalities and exploitation at a SRS, if agents discount future labour.

Theorem 17 Let $1>\rho$. Let $\left(p, w^{\prime}\right)$ be a SRS for $E\left(\Omega_{0}\right)$ with $\pi^{\prime}=(1-\rho) / \rho$, all t. Then:

(i) for all $\nu, \mu \in \mathcal{N}$, if $p^{\prime} \omega_{0}^{\mu}<\frac{p^{\prime} b \rho}{(1-\rho)}$, then $V\left(\omega_{0}^{\nu}\right)<V\left(\omega_{0}^{\mu}\right)$ if and only if $p^{\prime} \omega_{0}^{\nu}>p^{\prime} \omega_{0}^{\mu}$, where $p^{\prime} \equiv \frac{p}{w^{\prime}}$;

(ii) There is a constant $k^{\nu}$ such that $\Lambda_{t}^{\nu}-v b=k^{\nu}$ all $t, \nu$.

Proof. Part (i). Directly from Theorem 16, since $V\left(\omega_{0}^{\nu}\right)=0$ if and only if $p^{\prime} \omega_{0}^{\nu} \geq p^{\prime} b / \pi^{\prime} ;$ while if $V\left(\omega_{0}^{\nu}\right)>0$, then $V\left(\omega_{0}^{\nu}\right)-V\left(\omega_{0}^{\mu}\right)=\left(1-\rho^{T}\right)\left[p^{\prime} \omega_{0}^{\mu}-\right.$ $\left.p^{\prime} \omega_{0}^{\nu}\right] / \rho$ when $T$ is finite, and $V\left(\omega_{0}^{\nu}\right)-V\left(\omega_{0}^{\mu}\right)=\left[p^{\prime} \omega_{0}^{\mu}-p^{\prime} \omega_{0}^{\nu}\right] / \rho$ if $T \rightarrow \infty$.

Part (ii). Straightforward, given Proposition 11. 
Theorems 16 and 17 complete the intertemporal generalisation of Roemer's theory: the dynamic economy with discounting displays the same pattern of $W P$ and $W L$ exploitation as the $T$-fold repetition of the static economy, and both $W P$ and $W L$ exploitation are persistent. Moreover, unlike in the static model, the introduction of time preference in the dynamic model clarifies that Roemer's interpretation of Marxian exploitation at the WL level as an objectivist measure of inequalities - 'the exploitation-welfare criterion' (Roemer [24]: 75) - and subjectivist neoclassical welfare inequalities are different in general: the former notion focuses on asset inequalities, which are independent of time preference, while the latter focuses on welfare inequalities, which depend on $\rho$. According to Theorems 16 and 17, the two views coincide at a SRS, but they are conceptually distinct.

\subsection{The property relations definition of exploitation}

The previous sections generalise Roemer's UE approach to exploitation to the dynamic context and show that his definition of Marxian exploitation is distinct from, but conceptually related to neoclassical welfare inequalities. This section generalises Roemer's [24] game-theoretic approach, which focuses on property relations, a more general concept than asset inequalities.

Let $\left(V^{1}, \ldots, V^{N}\right)$ be the agents' payoffs at the existing allocation: in this context, it is natural to consider $\left(V^{1}, \ldots, V^{N}\right)$ as $W L$ values. For instance, at an RS for $E\left(\Omega_{0}\right), V^{1}=-V\left(\omega_{0}^{1}\right), \ldots, V^{N}=-V\left(\omega_{0}^{N}\right)$. Let $P(\mathcal{N})$ be the power set of $\mathcal{N}$ and let $K: P(\mathcal{N}) \rightarrow \mathbb{R}_{+}$be a characteristic function which assigns to every coalition $\mathcal{J} \subseteq \mathcal{N}$ with $J$ agents an aggregate payoff $K(\mathcal{J})$ if it withdraws from the economy.

Definition 18 ([24]: 194-195) Coalition $\mathcal{J} \subseteq \mathcal{N}$ is exploited at allocation $\left(V^{1}, \ldots, V^{N}\right)$ with respect to alternative $K$ if and only if the complement to $\mathcal{J}, \mathcal{N}-\mathcal{J}=\mathcal{J}^{\prime}$, is in a relation of dominance to $\mathcal{J}$ and

(i) $\sum_{\nu \in \mathcal{J}} V^{\nu}<K(\mathcal{J})$,

(ii) $\sum_{\nu \in \mathcal{J}^{\prime}} V^{\nu}>K\left(\mathcal{J}^{\prime}\right)$.

Definition 18 captures various kinds of exploitation, including Marxian exploitation, by specifying different hypothetically feasible alternatives. The concept of exploitation is related to the core of an economy: the set of nonexploitative allocations coincides with the core of the game described by $K$ (Roemer [24], Theorem 7.1: 198). The precise definition of exploitation 
depends on the function $K$. A coalition is feudally exploited at an allocation if it can improve by withdrawing from society with its own endowments and arranging production on its own. In $E\left(\Omega_{0}\right)$, the set of feudally nonexploitative allocations coincides with the private ownership core (POC). Formally, a coalition $\mathcal{J}$ is viable if it has enough assets to reproduce itself if it secedes from the parent economy (Roemer [24]: 45-49).

Definition 19 A coalition $\mathcal{J} \subseteq \mathcal{N}$ is viable if $\sum_{\nu \in \mathcal{J}} \omega_{0}^{\nu} \geqq J A(1-A)^{-1} b$.

A reproducible allocation is a profile of (not necessarily optimal) actions of all agents in $E\left(\Omega_{0}\right)$, that satisfy the feasibility and reproducibility constraints.

Definition 20 A reproducible allocation (RA) for $E\left(\Omega_{0}\right)$ is a profile of actions $\xi^{\nu}=\left(x^{\nu}, y^{\nu}, \gamma^{\nu}, s^{\nu}\right)$ for all $\nu$, such that

1. $L x_{t}^{\nu}+\gamma_{t}^{\nu} \leqq 1$, all $\nu, t$

2. $A\left(x_{t}+y_{t}\right) \leqq \omega_{t}$ all $t$;

3. $\left(x_{t}+y_{t}\right) \geqq A\left(x_{t}+y_{t}\right)+N b+s_{t}$, all $t$;

4. $\omega_{t+1}=\omega_{t}+s_{t}$, all $t$;

5. $\omega_{T} \geqq \omega_{0}^{\nu}$.

A viable coalition $\mathcal{J}$ can block a $\mathrm{RA}\left(\xi^{\nu}\right)_{\nu \in \mathcal{N}}$ if there is another RA for the smaller economy that yields higher welfare to its members.

Definition 21 A viable coalition $\mathcal{J}$ can block a $R A\left(\xi^{\nu}\right)_{\nu \in \mathcal{N}}$ if there is a profile $\left(\xi^{\prime 1}, \ldots, \xi^{\prime J}\right)$ such that

1. $\sum_{t=0}^{T-1} \rho^{t} \Lambda_{t}^{\nu}<\sum_{t=0}^{T-1} \rho^{t} \Lambda_{t}^{\nu}$, all $\nu \in \mathcal{J}$;

2. $A \sum_{\nu \in \mathcal{J}} x_{t}^{\prime \nu} \leqq \sum_{\nu \in \mathcal{J}} \omega_{t}^{\nu}$, all $t$;

3. $(1-A) \sum_{\nu \in \mathcal{J}} x_{t}^{\prime \nu}=J b+\sum_{\nu \in \mathcal{J}} s_{t}^{\prime \nu}$, all $t$;

4. $\sum_{\nu \in \mathcal{J}} \omega_{t+1}^{\nu}=\sum_{\nu \in \mathcal{J}} \omega_{t}^{\nu}+\sum_{\nu \in \mathcal{J}} s_{t}^{\prime \nu}$, all $t$;

5. $\sum_{\nu \in \mathcal{J}} \omega_{T}^{\nu} \geqq \sum_{\nu \in \mathcal{J}} \omega_{0}^{\nu}$.

The POC of $E\left(\Omega_{0}\right)$ is the set of RAs which no coalition can block. Theorem 22 proves the absence of feudal exploitation in $E\left(\Omega_{0}\right)$.

Theorem 22 Let $\rho \leqq 1$. Any IRS of $E\left(\Omega_{0}\right)$ lies in its private ownership core and thus displays no feudal exploitation. 
Proof. 1. If $\pi_{t}=0$, all $t$, the result is trivial. Hence, assume $\pi_{0}>0$.

2. Suppose that there is $\mathcal{J} \subseteq \mathcal{N}$ that can block the IRS. By Definition 21(1), no pure capitalist can belong to $\mathcal{J}$; thus, by Lemma 9 and Proposition 11, at an IRS $(p, \mathbf{w}), \pi_{t} \frac{p}{w_{t}} \omega_{0}^{\nu}=\frac{p}{w_{t}} b-\Lambda_{t}^{\nu}$ all $t$ and all $\nu \in \mathcal{J}$. Summing over $\nu$ $\in \mathcal{J}$ and $t, \sum_{t=0}^{T-1} \rho^{t} \pi_{t} \frac{p}{w_{t}} \sum_{\nu \in \mathcal{J}} \omega_{0}^{\nu}=\sum_{t=0}^{T-1} \rho^{t} J \frac{p}{w_{t}} b-\sum_{t=0}^{T-1} \rho^{t} \sum_{\nu \in \mathcal{J}} \Lambda_{t}^{\nu}$. By Proposition 12, $\sum_{t=0}^{T-1} \rho^{t} \pi_{t} \frac{p}{w_{t}} \sum_{\nu \in \mathcal{J}} \omega_{0}^{\nu}=\left[\left(1+\pi_{0}\right) \frac{p}{w_{0}}-\rho^{T-1} \frac{p}{w_{T-1}}\right] \sum_{\nu \in \mathcal{J}} \omega_{0}^{\nu}$.

3. If $\mathcal{J}$ can block the IRS, multiplying Definition 21(3) by $\rho^{t} v$ and summing over $t, \sum_{t=0}^{T-1} \rho^{t} \sum_{\nu \in \mathcal{J}} \Lambda_{t}^{\nu \nu}=\sum_{t=0}^{T-1} \rho^{t} J v b+\sum_{t=0}^{T-1} \rho^{t} v \sum_{\nu \in \mathcal{J}} s_{t}^{\nu}$. By Definition 21(1) and step 2: $\quad \sum_{t=0}^{T-1} \rho^{t} J\left(\frac{p}{w_{t}}-v\right) b-\sum_{t=0}^{T-1} \rho^{t} v \sum_{\nu \in \mathcal{J}} s_{t}^{\nu}>$ $\left[\left(1+\pi_{0}\right) \frac{p}{w_{0}}-\rho^{T-1} \frac{p}{w_{T-1}}\right] \sum_{\nu \in \mathcal{J}} \omega_{0}^{\nu}$.

4. If $\mathcal{J}$ can block the IRS, by Definition 21(2)-(3), $A(1-A)^{-1}(J b+$ $\left.\sum_{\nu \in \mathcal{J}} s_{t}^{\nu}\right) \leqq \sum_{\nu \in \mathcal{J}} \omega_{t}^{\nu}$ all $t$; multiplying both sides by $\rho^{t} \pi_{t} \frac{p}{w_{t}}, \rho^{t}\left(\frac{p}{w_{t}}-v\right) J b-$ $\rho^{t} v \sum_{\nu \in \mathcal{J}} s_{t}^{\nu} \leqq \rho^{t} \pi_{t} \frac{p}{w_{t}} \sum_{\nu \in \mathcal{J}} \omega_{t}^{\nu}-\rho^{t} \frac{p}{w_{t}} \sum_{\nu \in \mathcal{J}} s_{t}^{\nu}$ all $t$. Summing over $t$, by Definition 21(4), the latter expression becomes $\sum_{t=0}^{T-1} \rho^{t}\left(\frac{p}{w_{t}}-v\right) J b-\sum_{t=0}^{T-1} \rho^{t} v \sum_{\nu \in \mathcal{J}} s_{t}^{\nu} \leqq$ $\sum_{t=0}^{T-1} \rho^{t}\left[\left(1+\pi_{t}\right) \frac{p}{w_{t}} \sum_{\nu \in \mathcal{J}} \omega_{t}^{\nu}-\frac{p}{w_{t}} \sum_{\nu \in \mathcal{J}} \omega_{t+1}^{\nu}\right]$. Then, using $\rho\left(1+\pi_{t+1}\right) \frac{p}{w_{t}+1}=$ $\frac{p}{w_{t}}$ all $t, \sum_{t=0}^{T-1} \rho^{t}\left(\frac{p}{w_{t}}-v\right) J b-\sum_{t=0}^{T-1} \rho^{t} v \sum_{\nu \in \mathcal{J}} s_{t}^{\nu} \leqq\left(1+\pi_{0}\right) \frac{p}{w_{0}} \sum_{\nu \in \mathcal{J}} \omega_{0}^{\nu}-$ $\rho^{T-1} \frac{p}{w_{T-1}} \sum_{\nu \in \mathcal{J}} \omega_{T}^{\nu}$.

5. The latter inequality and the inequality in step 3 can both hold only if $\sum_{\nu \in \mathcal{J}} \omega_{T}^{\nu}<\sum_{\nu \in \mathcal{J}} \omega_{0}^{\nu}$, which contradicts Definition 21(5).

In Roemer's interpretation of historical materialism as predicting the progressive disappearance of various forms of exploitation, Theorem 22 proves that capitalist relations of production eliminate feudal exploitation. It also clarifies the neoclassical claim concerning the absence of exploitation in a competitive economy: there is no feudal exploitation (Roemer [24]: 205-8).

A different specification of $K$ is necessary to define capitalist exploitation. Let $\omega_{0}^{\mathcal{J}} \equiv \frac{J}{N} \omega_{0}^{\nu}$ be coalition $\mathcal{J}$ 's per-capita share of aggregate initial assets. Given the linear technology, all coalitions are viable if they withdraw with $\omega_{0}^{\mathcal{J}}$. Then, a coalition can communally block a RA if it can increase the welfare of its members by withdrawing with $\omega_{0}^{\mathcal{J}}$.

Definition 23 A coalition $\mathcal{J}$ can communally block a $R A\left(\xi^{\nu}\right)_{\nu \in \mathcal{N}}$ if there is a profile of vectors $\left(\xi^{\prime 1}, \ldots, \xi^{\prime J}\right)$ such that

1. $\sum_{t=0}^{T-1} \rho^{t} \Lambda_{t}^{\prime \nu}<\sum_{t=0}^{T-1} \rho^{t} \Lambda_{t}^{\nu}$, all $\nu \in \mathcal{J}$;

2. $A \sum_{\nu \in \mathcal{J}} x_{t}^{\prime \nu} \leqq \omega_{t}^{\mathcal{J}}$, all $t$;

3. $(1-A) \sum_{\nu \in \mathcal{J}} x_{t}^{\prime \nu}=J b+\sum_{\nu \in \mathcal{J}} s_{t}^{\prime \nu}$, all $t$;

4. $\omega_{t+1}^{\mathcal{J}}=\omega_{t}^{\mathcal{J}}+\sum_{\nu \in \mathcal{J}} s_{t}^{\prime \nu}$, all $t$ 
5. $\omega_{T}^{\mathcal{J}} \geqq \omega_{0}^{\mathcal{J}}$.

The communal core of $E\left(\Omega_{0}\right)$ is the set of RAs which no coalition can communally block; a coalition is capitalistically exploited if it can communally block the RA; and a RA is capitalist non-exploitative if it lies in the communal core of the economy. Theorem 24 proves that Marxian exploitation and capitalist exploitation coincide in $E\left(\Omega_{0}\right)$ at an IRS.

Theorem 24 Let $\rho \leqq 1$. At an IRS, a coalition is WL Marxian exploited if and only if it is capitalistically exploited.

Proof. If a coalition $\mathcal{J}$ is Marxian exploited, $\sum_{t=0}^{T-1}\left(\sum_{\nu \in \mathcal{J}} \Lambda_{t}^{\nu}-J v b\right)>0$. But then by Proposition 15, at an IRS $\sum_{t=0}^{T-1} \rho^{t}\left(\sum_{\nu \in \mathcal{J}} \Lambda_{t}^{\nu}-J v b\right)>0$, and $\mathcal{J}$ can communally block the allocation. The converse is proved similarly.

Theorem 24 suggests that Marxian exploitation can be seen as a special case of Roemer's Definition 18 in a linear economy with labour-minimising agents. The property-relation definition (which can be applied to a general set of economies; Roemer [24], chapter 7) would then be a generalisation of Marx's theory that captures its essential normative content.

\subsection{Power and the persistence of exploitation}

The previous results provide a complete dynamic generalisation of Roemer's distributive approach, and thus may be seen as confirming Roemer's key theoretical insight that exploitation can be reduced to a concern for asset inequalities. This section raises some doubts on this conclusion. For DOPA is not necessary and sufficient to generate persistent exploitation.

Theorem 25 shows that if agents do not discount the future, profits and the UE of labour tend to decrease over time.

Theorem 25 Let $\rho=1$. Let $(p, \mathbf{w})$ be an IRS for $E\left(\Omega_{0}\right)$ with $\pi_{0}>0$. Then (i) $\pi_{t}>\pi_{t+1}$, all t. Moreover, (ii) for all $\nu \in \mathcal{N}$ such that $\Lambda^{\nu}>0$ for all $\xi^{\nu} \in \mathcal{O}(p, \mathbf{w})$, at all $t$ : if $W_{t}^{\nu}<W_{t}^{*}$ then $\Lambda_{t}^{\nu}>\Lambda_{t+1}^{\nu}$, if $W_{t}=W_{t}^{*}$ then $\Lambda_{t}^{\nu}=\Lambda_{t+1}^{\nu}$, and if $W_{t}>W_{t}^{*}$ then $\Lambda_{t}^{\nu}<\Lambda_{t+1}^{\nu}$.

Proof. Part (i). The result follows noting that $\frac{p}{w_{t}}$ is a continuous, increasing function of $\pi_{t}$, all $t$, while by Proposition $12, \frac{p}{w_{t}}>\frac{p}{w_{t+1}}$ all $t$. 
Part (ii). By Proposition 15 if $W_{t}^{\nu}=W_{t}^{*}$ then $\Lambda_{t}^{\nu}=\Lambda_{t+1}^{\nu}=v b$, all t. By Proposition 11, $\Lambda_{t+1}^{\nu}-\Lambda_{t}^{\nu}=\left(\frac{p}{w_{t+1}}-\frac{p}{w_{t}}\right) b+\left(\pi_{t} \frac{p}{w_{t}}-\pi_{t+1} \frac{p}{w_{t+1}}\right) \omega_{0}^{\nu}$ or, equivalently, $\Lambda_{t+1}^{\nu}-\Lambda_{t}^{\nu}=\left(\frac{p}{w_{t+1}}-\frac{p}{w_{t}}\right) b+\left(\pi_{t}-\frac{\pi_{t+1}}{1+\pi_{t+1}}\right) \frac{p}{w_{t}} \omega_{0}^{\nu}$. Therefore the result follows from part $(i)$ and the monotonicity of the right hand side of the latter expression in $W_{t}^{\nu}$.

Theorem 25 is rather counterintuitive. In the equilibrium that preserves the exploitation structure of the competitive economy, profits and $W P$ exploitation decrease over time: $W P$ exploiters work more while $W P$ exploited agents work less, even if neither accumulates. The simple possibility of saving implies a decrease in the dispersion of agents' labour times around $v b$, due to the decrease in profits.

Theorem 26 strengthens these conclusions by looking at the long-run behaviour of the economy.

Theorem 26 Let $\rho=1$ and $T \rightarrow \infty$. Let $(p, \mathbf{w})$ be an IRS for $E\left(\Omega_{0}\right)$ with $\pi_{0}>0$. Then $\Lambda_{t}^{\nu} \rightarrow v b$ and $\frac{p}{w_{t}} \omega_{t}^{\nu} \rightarrow v \omega_{0}^{\nu}$, all $\nu$, as $t \rightarrow \infty$.

Proof. By $\pi_{t}=\left[\frac{p}{w_{t}}(1-A)-L\right] / \frac{p}{w_{t}} A$ and Proposition 12, if $\rho=1$ then $\frac{p}{w_{t+1}}=\frac{p}{w_{t}} A+L$ at IRS, thus $\frac{p}{w_{t}}=\left[\frac{p}{w_{0}}-L(1-A)^{-1}\right] A^{t}+L(1-A)^{-1}$, which implies by $A \in(0,1)$ that $\frac{p}{w_{t}} \rightarrow v$ and $\pi_{t} \rightarrow 0$ as $t \rightarrow \infty$.

Theorem 26 completes the analysis. The previous sections extend Roemer's theory to the intertemporal context, but the key results crucially depend on the assumption that $\rho<1$. If $\rho=1$, at any SRS, Proposition 12 implies zero profits leading to a non-exploitative allocation. Theorems 25 and 26 generalise this conclusion. In the equilibrium which preserves DOPA and the exploitation structure of the economy, profits and WP exploitation decrease over time and tend to disappear in the long run, even if capital scarcity persists (unlike in accumulation models, such as Devine and Dymsky [4]). ${ }^{9}$

This conclusion is robust. The above results extend and generalise analogous conclusions by Veneziani [34], [35] in dynamic subsistence economies. Moreover, Veneziani and Yoshihara [37] have shown that, whenever $\rho=1$,

\footnotetext{
${ }^{9}$ Okishio [21] also shows that in a dynamic capitalist economy with neither population growth nor technical change, competition among capitalists may drive profits and exploitation to zero. According to Okishio [21], this profit squeeze derives from the increase in the real wage rate due to capital accumulation. Okishio's [21] results, however, are based on simulation methods and only hold for a specific choice of parameters.
} 
$W P$ exploitation also tends to disappear in more general dynamic general equilibrium models with convex technologies and standard utility functions defined over consumption and leisure. The question, then, concerns the implications of these results for exploitation theory.

Proposition 15, and Theorems 24 and 26 can be interpreted as identifying asset inequalities and a strictly positive rate of time preference as the necessary and sufficient conditions for the persistence of exploitation in a neoclassical dynamic framework. This questions Roemer's claim that all the relevant normative intuitions about exploitation are captured by DOPA.

In fact, as noted above, although Roemer's argument about the focal, indeed exclusive relevance of asset inequalities is normative in nature, it crucially rests on a positive claim that 'differential distribution of property and competitive markets are sufficient institutions to generate an exploitation phenomenon, under the simplest possible assumptions' (Roemer [24]: 43). This suggests that the Marxian concept of exploitation can be reduced to an asset-based approach, and provides the foundations for Definition 18. As Skillman ([31]: 311) aptly noted, 'the legitimacy of Roemer's reformulation depends in large part on the validity of his claims concerning the role of DOPA in capitalist exploitation'.

By significantly qualifying Roemer's positive claim, Proposition 15 and Theorems 24 and 26 raise some doubts on Definition 18 both per se and as a generalisation of Marx's theory. For they prove that at a RS where no agent accumulates and capital scarcity persists, DOPA is necessary to generate UE exploitation, but it is not sufficient for it to persist. Thus, the persistence of DOPA per se is not a sufficient statistic of the unfairness of labour/capital relations (and more generally, of a society). Something else is indispensable to guarantee the persistence of exploitation, which would be normatively at least as important as DOPA itself. Definition 18 may be seen as incorporating a different moral concern, rather than as a generalisation of Marx's definition. More generally, the question arises whether DOPA should be a basic moral concern, both in itself and in a theory of exploitation, or rather a different role of DOPA should be stressed as a causally primary, but normatively secondary wrong.

To be sure, it may be argued that the above analysis shows that exploitation is persistent, provided agents discount the future. This objection is not entirely compelling. As Veneziani [35] has argued, Roemer's key argument is a logical claim about the sufficiency of DOPA - and of the key institutional features of capitalist economies (i.e. competitive markets) - to generate ex- 
ploitation. The specific value of a parameter of the agents' utility function should not be relevant at this level of abstraction. Moreover, whether agents do, or do not, display impatient time preferences is a purely empirical issue, and one which has a priori little to do with DOPA or with the fundamental features of a capitalist economy.

Finally, a theoretical argument that crucially relies on time preference seems at odds with the core intuitions of UE exploitation theory which emphasise the structural features of capitalist economies. As Roemer ([26]: 60ff) himself notes, the normative relevance of a theory of exploitation critically relying on such exogenous factors would be rather unclear. This is particularly relevant in our model because, by Theorems 16 and 17, both the persistence and the magnitude of exploitation and welfare inequalities depend on time preference. Given the positive relation between the profit rate, and welfare inequalities and exploitation, the higher $\rho$, the lower the equilibrium profit rate, and the lower UE exploitation, ceteris paribus.

In summary, the above results provide a robust criticism of Roemer's core claim that DOPA is the fundamental cause of exploitation. This claim crucially depends on very restrictive assumptions, such as the impossibility of savings. If savings are allowed, DOPA is necessary but not sufficient to generate persistent exploitation, and an emphasis on asset inequalities while exploitation disappears seems misplaced. Therefore, the intertemporal model raises serious doubts on the claim that exploitation theory can be reduced to a form of resource egalitarianism. ${ }^{10}$

It is certainly possible, and interesting, to investigate some mechanisms that guarantee the persistent abundance of labour in a capitalist economy. Skillman [31] and Okishio [21], for example, suggest that a dynamic model including growth in the labour force and/or labour-saving technical change might provide micro-foundations to persistent exploitation in a Marxian framework. This would be consistent with Marx's own approach, which

\footnotetext{
${ }^{10}$ It might be objected that $W L$ exploitation does not disappear, even if $\rho=1$, and the relationship between initial wealth and $W L$ exploitation status is preserved. Thus, from a mathematical viewpoint, the model may be interpreted as a generalisation of Roemer's theory under the $W L$ definition. Yet, this does not affect our main conclusions. First, given the theoretical relevance of the WP definition, Marxian exploitation should arguably be micro-founded as a persistent WP phenomenon. Second, not only is the tendential disappearance of WP exploitation disturbing per se; it also implies that ceteris paribus, $W L$ exploitation, too, is lower in the dynamic model with agents living for $T$ periods than in the $T$-fold iteration of the static model.
} 
focused on (long-run) equilibria with unemployment due to labour-saving technical progress, whereas the analysis above focuses on the neoclassical full employment equilibrium, which is not the standard feature of capitalist economy for Marx even if $\rho=1$. However, even if exploitation could be proved to be persistent under those assumptions, it is unclear whether our main conclusions would change. For DOPA and competitive markets would still be insufficient to yield persistent WP exploitation.

\section{Conclusions}

This paper has presented and extended some recent work in exploitation theory. Three main conclusions can be drawn from our analysis. First, the notion of exploitation as the unequal exchange of labour is logically coherent and can be meaningfully defined in economies that are significantly more general than those usually analysed in mathematical Marxian economics. The model set up in section 2 allows for choice of technique, joint production, heterogeneous intertemporally optimising agents with general preferences over consumption and leisure, and different endowments of physical and human capital. Although the model incorporates some features that are not standard in neoclassical theory, such as the time structure of production and the reproducibility of all capital goods, the dynamic equilibrium notion is conceptually cognate to standard notions used in optimal growth models.

Second, the normative foundations of UE exploitation can be analysed by adopting the axiomatic method, and an extension of the 'New Interpretation' (Duménil [5], [6]; Foley [11], [12]) is the only definition (among the main approaches in the literature) satisfying a number of weak and desirable properties in the general economies analysed in this paper.

Third, exploitation cannot be reduced to a focus on asset inequalities: even if capital scarcity and DOPA persist, in dynamic subsistence economies UE exploitation tends to disappear. A concern for power, dominance, or coercion is an integral part of the notion of exploitation and can contribute to mitigate the 'distributive bias' of normative economics.

It is important to stress that this paper does not provide the final word on exploitation theory. It suggests that UE exploitation can be analysed rigorously with the standard tools of normative economics and social choice theory, and that a logically coherent and normatively interesting notion of exploitation can be formulated in general economies. Yet many important is- 
sues remain unanswered which represent promising lines for further research. In the rest of this section, we discuss some of them.

First, the axiomatic analysis in section 3 is based on the 'contribution view' of exploitation theory: exploitative relations are characterised by systematic differences between the amount of effective labour that agents contribute to the economy and the labour received by them. As noted by an anonymous referee, however, one may emphasise the 'welfare view' of exploitation theory, whereby the normative relevance of UE exploitation derives from the fact that income and labour time are fundamental determinants of individual well-being freedom (e.g. Rawls [22]). If one adopted the welfare view, then perhaps both the definitions of exploitation in section 3.1 and the main domain axiom $\mathbf{L E}$ should be expressed in terms of labour time.

Second, section 4 raises the issue of the appropriate definition of exploitation and in particular the role of distributional and power-related concerns in exploitation theory. Our results suggest that, contrary to Roemer's claim, Marxian exploitation cannot be reduced to asset inequalities and the resulting welfare inequalities. This raises two sets of issues.

At a normative level, as Veneziani [35] has argued, the dominance condition in Definition 18, is not just necessary 'to rule out some bizarre examples' (Roemer [24]: 195): asymmetric relations of power, or dominance should play a definitional role in a theory of exploitation as a social relation in competitive economies. Exploitation should thus be conceived of as involving both the outcome and the structure of the interaction between agents, as it diagnoses the process through which 'certain inequalities in incomes are generated by inequalities in rights and powers over productive resources: the inequalities occur, in part at least, through the ways in which the exploiters, by virtue of their exclusionary rights and powers over resources, are able to appropriate labour effort of the exploited' (Wright [39]: 1563).

At a positive level, the question arises as to the key determinants of the persistence of exploitation in capitalist economies. Arguably, here too, a focus on power, or dominance, may contribute to a more satisfactory explanation of persistent exploitative relations based on the structural features of capitalist economies. As Devine and Dymski [4] noted, two implicit assumptions are necessary in Roemer's theory in order to generate persistent exploitation: capital scarcity and exogenous labour intensity. The former disappears when capital accumulation is introduced, the latter is violated when labour contracts are incomplete. Without complete contracts, exploitative relations may not arise even in a static setting because of the 
profit-squeeze caused by the lack of labour-discipline in production. ${ }^{11}$ Building on this point, Yoshihara [40] integrates incomplete labour contracts into the standard general equilibrium framework of Marxian exploitation theory, and shows that the degree of exploitation is related to the strength of the power relationship which is in turn affected by the degree of asset inequalities: poor agents are forced to provide a higher level of labour intensity per wage rate than wealthier agents.

Given its concern with power and the emphasis on the role of physical assets in explaining hierarchical relations and the existence of firms, the property rights theory of the firm (Hart [13]) may also provide an interesting theoretical framework to analyse exploitative relations which goes beyond purely distributive views and is consistent with the idea that asset inequalities are causally primary, but normatively secondary.

\section{References}

[1] Baigent, N., 1981. Decompositions of Minimal Liberalism. Economics Letters 7, 29-32.

[2] Bowles, S., Gintis, H., 1990. Contested Exchange: New Microfoundations of the Political Economy of Capitalism. Politics and Society 18, $165-222$.

[3] Cohen, G.A., 1995. Self-ownership, freedom and equality. Cambridge University Press, Cambridge.

[4] Devine, J., Dymski, G., 1991. Roemer's 'General' Theory of Exploitation is a Special Case. Economics and Philosophy 7, 235-275.

[5] Duménil, G., 1980. De la Valeur aux Prix de Production. Economica, Paris.

[6] Duménil, G., 1984. The So-Called 'Transformation Problem' Revisited: A Brief Comment. Journal of Economic Theory 33, 340-348.

[7] Duménil, G., Foley, D.K., Lévy, D., 2009. A Note on the Formal Treatment of Exploitation in a Model with Heterogeneous Labor. Metroeconomica $60,560-567$.

\footnotetext{
${ }^{11}$ This point is also raised by Bowles and Gintis [2]
} 
[8] Flaschel, P., 1983. Actual Labor Values in a General Model of Production. Econometrica 51, 435-454.

[9] Fleurbaey, M., 1996. Théories Économiques de la Justice. Economica, Paris.

[10] Fleurbaey, M., 2013. The facets of exploitation. Journal of Theoretical Politics, forthcoming.

[11] Foley, D.K., 1982. The Value of Money, the Value of labour Power, and the Marxian Transformation Problem. Review of Radical Political Economics 14, 37-47.

[12] Foley, D.K., 1982. Realization and Accumulation in a Marxian Model of the Circuit of Capital. Journal of Economic Theory 28, 300-319.

[13] Hart, O. D., 1995. Firms, Contracts, and Financial Structure. Oxford University Press, Oxford.

[14] Krause, U., 1982. Heterogeneous Labour and the Fundamental Marxian Theorem. Review of Economic Studies 48, 173-178.

[15] Maniquet, F., 2002. A study of proportionality and robustness in economies with a commonly owned technology. Review of Economic Design $7,1-15$.

[16] Matsuo, T., 2008. Profit, Surplus Product, Exploitation and Less than Maximized Utility. Metroeconomica 59, 249-265.

[17] Morishima, M., 1969. Theory of Economic Growth. Oxford University Press, Oxford.

[18] Morishima, M., 1973. Marx's Economics, Cambridge University Press, Cambridge.

[19] Morishima, M., 1974. Marx in the Light of Modern Economic Theory. Econometrica 42, 611-632.

[20] Okishio, N., 1963. A mathematical note on Marxian Theorems, Weltwirtschaftliches Archiv 91, 287-299. 
[21] Okishio, N., 2000. Competition and Production Prices. Cambridge Journal of Economics 25, 493-501.

[22] Rawls, J., 1971. A Theory of Justice, Harvard University Press, Cambridge, MA.

[23] Roemer, J.E., 1981. Analytical foundations of Marxian economic theory. Harvard University Press, Cambridge, MA.

[24] Roemer, J.E., 1982. A General Theory of Exploitation and Class. Harvard University Press, Cambridge, MA.

[25] Roemer, J.E., 1982. New directions in the Marxian theory of exploitation and classes. Politics and Society 11, 253-87.

[26] Roemer, J.E., 1988. Free to Lose. Cambridge: Harvard University Press.

[27] Roemer, J.E., 1989. Marxism and Contemporary Social Science. Review of Social Economy 47, 377-391.

[28] Roemer, J.E., 1994. Egalitarian Perspectives: Essays in Philosophical Economics. Cambridge University Press, Cambridge.

[29] Roemer, J.E., 2010. Kantian equilibrium. Scandinavian Journal of Economics 112, 1-24.

[30] Roemer, J.E., Silvestre, J.E., 1993. The proportional solution for economies with both private and public ownership. Journal of Economic Theory 59, 426-444.

[31] Skillman, G.L., 1995. Ne Hic Saltaveris: the Marxian Theory of Exploitation after Roemer. Economics and Philosophy 11, 309-331.

[32] Steiner, H. 2013. Liberalism, neutrality and exploitation. Politics, Philosophy \& Economics, forthcoming.

[33] van Donselaar, G. 2009. The Right to Exploit. Oxford University Press, Oxford.

[34] Veneziani, R., 2007. Exploitation and Time. Journal of Economic Theory $132,189-207$. 
[35] Veneziani, R., 2013. Exploitation, Inequality, and Power. Journal of Theoretical Politics, forthcoming.

[36] Veneziani, R., Yoshihara, N., 2013. Exploitation in economies with heterogeneous preferences, skills and assets: An axiomatic approach. Journal of Theoretical Politics, forthcoming.

[37] Veneziani, R., Yoshihara, N., 2012. Globalisation and inequalities, Mimeo, Queen Mary University of London, and IER, Hitotsubashi University.

[38] Vrousalis, N., 2013. Exploitation, Vulnerability, and Social Domination. Philosophy and Public Affairs 41, 131-157.

[39] Wright, E.O., 2000. Class, Exploitation, and Economic Rents: Reflections on Sorensen's 'Sounder Basis'. The American Journal of Sociology 105, 1559-1571.

[40] Yoshihara, N., 1998. Wealth, Exploitation, and Labor Discipline in the Contemporary Capitalist Economy. Metroeconomica 49, 23-61.

[41] Yoshihara, N., 2010. Class and Exploitation in General Convex Cone Economies. Journal of Economic Behavior \& Organization 75, 281-296.

[42] Yoshihara, N., Veneziani, R., 2009. Exploitation as the Unequal Exchange of Labour: An Axiomatic Approach. Working paper N.655, Queen Mary University of London.

[43] Yoshihara, N., Veneziani, R., 2011. Strong Subjectivism in the Theory of Exploitation: A Critique. Metroeconomica 62, 53-68.

[44] Yoshihara, N., Veneziani, R., 2012. Exploitation of Labour and Exploitation of Commodities: a 'New Interpretation.' Review of Radical Political Economics, forthcoming.

[45] Ypi, L., 2010. On the Confusion between Ideal and Non-ideal in Recent Debates on Global Justice. Political Studies 58, 536-555. 